\title{
Quantum-kinetic theory of photocurrent generation via direct and phonon-mediated optical transitions
}

\author{
U. Aeberhard* \\ IEK-5 Photovoltaik, Forschungszentrum Jülich, D-52425 Jülich, Germany
}

(Received 31 December 2010; revised manuscript received 3 April 2011; published 29 July 2011)

\begin{abstract}
A quantum kinetic theory of direct and phonon-mediated indirect optical transitions is developed within the framework of the nonequilibrium Green's function formalism. After validation against the standard Fermi golden rule approach in the bulk case, it is used in the simulation of photocurrent generation in ultrathin crystalline silicon $p-i-n$ junction devices.
\end{abstract}

DOI: 10.1103/PhysRevB.84.035454

PACS number(s): $72.40 .+\mathrm{w}, 78.20 . \mathrm{Bh}$

\section{INTRODUCTION}

Quantum effects in semiconductor nanostructures are widely exploited in optoelectronic devices such as light emitting diodes or lasers. With the increasing demand of renewable energy supply, high efficiency photovoltaic devices were proposed, which also make use of semiconductor nanostructures. ${ }^{1,2}$ However, these devices are based not on the standard III-V direct band gap materials conventionally used in optoelectronics but on silicon, which is most common in solar cell applications. It was shown that even though the suppression of direct optical transitions is somewhat relaxed in low-dimensional silicon structures, it is the phonon-assisted indirect processes that dominate the optical response, ${ }^{3}$ and thus a suitable theoretical description of the photovoltaic behavior needs to include these processes responsible for energy and momentum transfer, in addition to the general requirements of a quantum photovoltaic device, which were addressed in Ref. 4. As an extension of this previous work, the present paper presents a microscopic description of phonon-mediated optical transitions in indirect semiconductor nanostructures, based on the nonequilibrium Green's function (NEGF) formalism and thus compatible with advanced quantum transport theories.

The paper is organized as follows. After the derivation of the general expressions for optoelectronic rates within the nonequilibrium Green's function theory, these are applied to the case of a direct gap bulk semiconductor and compared to the standard Fermi golden-rule (FGR) result. Next, the description of phonon-mediated indirect transitions within the NEGF formalism is discussed and the resulting expression for the generation rate again compared to the FGR, both in an analytical approximation and a numerical computation for a simple effective mass model of bulk silicon. Finally, the theory is extended to thin films in order to model photocurrent generation in ultrathin indirect gap semiconductor $p-i-n$ junctions, which form important building blocks of future nanophotovoltaic devices.

\section{NEGF THEORY}

In order to be able to describe both optical transitions and inelastic quantum transport in semiconductor nanostructures within the same microscopic picture, a theoretical framework based on the NEGF formalism was developed in Ref. 4. Before considering the specific case of photogeneration in indirect semiconductors, the aspects of the theory related to interband transitions will be outlined below in a general formulation, which relies on the extensive work on similar systems investigated with focus on their light-emitting and lasing properties. ${ }^{5-12}$

\section{A. Interband generation and recombination rates}

The macroscopic equation of motion for a photovoltaic system is the continuity equation for the charge carrier density

$$
\partial_{t} \rho_{c}(\mathbf{r})+\nabla \cdot \mathbf{j}_{c}(\mathbf{r})=\mathcal{G}_{c}(\mathbf{r})-\mathcal{R}_{c}(\mathbf{r}), \quad c=e, h,
$$

where $\rho_{c}$ and $j_{c}$ are charge carrier and particle current density, respectively, $\mathcal{G}_{c}$ the generation rate and $\mathcal{R}_{c}$ the recombination rate of carrier species $c .{ }^{13}$ The microscopic version of this equation in terms of NEGF corresponds to ${ }^{14,15}\left(\underline{1} \equiv\left\{\mathbf{r}_{1}, t_{1} \in\right.\right.$ C\})

$$
\begin{gathered}
\lim _{2 \rightarrow 1}\left\{i \hbar\left(\frac{\partial}{\partial t_{1}}+\frac{\partial}{\partial t_{2}}\right) G(\underline{1}, \underline{2})+\left[H_{0}\left(\mathbf{r}_{1}\right)-H_{0}\left(\mathbf{r}_{2}\right)\right] G(\underline{1}, \underline{2})\right\} \\
\quad=\lim _{2 \rightarrow 1} \int_{\mathcal{C}} d 3[\Sigma(\underline{1}, \underline{3}) G(\underline{3}, \underline{2})-G(\underline{1}, \underline{3}) \Sigma(\underline{3}, \underline{2})],
\end{gathered}
$$

where $H_{0}$ is the Hamiltonian of the noninteracting electronic system, $G$ is the electronic nonequilibrium Green's function defined on the Keldysh contour $\mathcal{C}$, and $\Sigma$ is the self-energy encoding the interaction of the electronic system with phonons, photons, and itself, i.e., the scattering processes that may give rise to intra- or interband transitions. In steady state, (1) becomes

$$
\nabla \cdot \mathbf{j}_{c}(\mathbf{r})=\mathcal{G}_{c}(\mathbf{r})-\mathcal{R}_{c}(\mathbf{r}), \quad c=e, h .
$$

In the microscopic theory, the divergence of the electron (particle) current density corresponds to the limit $1 \rightarrow 2$ of the right-hand side in (2), ${ }^{16}$

$$
\begin{aligned}
\nabla \cdot \mathbf{j}(\mathbf{r})= & -2 \int \frac{d E}{2 \pi \hbar} \int d^{3} r^{\prime}\left[\Sigma^{R}\left(\mathbf{r}, \mathbf{r}^{\prime} ; E\right) G^{<}\left(\mathbf{r}^{\prime}, \mathbf{r} ; E\right)\right. \\
& +\Sigma^{<}\left(\mathbf{r}, \mathbf{r}^{\prime} ; E\right) G^{A}\left(\mathbf{r}^{\prime}, \mathbf{r} ; E\right)-G^{R}\left(\mathbf{r}, \mathbf{r}^{\prime} ; E\right) \\
& \left.\times \Sigma^{<}\left(\mathbf{r}^{\prime}, \mathbf{r} ; E\right)-G^{<}\left(\mathbf{r}, \mathbf{r}^{\prime} ; E\right) \Sigma^{A}\left(\mathbf{r}^{\prime}, \mathbf{r} ; E\right)\right] .
\end{aligned}
$$

If the energy integration is taken over the range of all bands connected by the interband scattering process, i.e., both valence and conduction bands, the resulting divergence should vanish, if the self-energies are chosen properly, ensuring the conservation of the overall current, which is nothing else than the corresponding formulation of the detailed balance requirement. If the integration is restricted to one of the bands 
(e.g., $B=$ valence band or conduction band), the above equation corresponds to the microscopic version of (3) and provides on the right-hand side the total local interband scattering rate,

$$
\begin{aligned}
R_{\mathrm{rad}}(\mathbf{r}) \equiv & \mathcal{R}(\mathbf{r})-\mathcal{G}(\mathbf{r}) \\
= & -2 \int_{B(\mathbf{r})} \frac{d E}{2 \pi \hbar} \int d^{3} r^{\prime}\left[\Sigma^{R}\left(\mathbf{r}, \mathbf{r}^{\prime} ; E\right) G^{<}\left(\mathbf{r}^{\prime}, \mathbf{r} ; E\right)\right. \\
& +\Sigma^{<}\left(\mathbf{r}, \mathbf{r}^{\prime} ; E\right) G^{A}\left(\mathbf{r}^{\prime}, \mathbf{r} ; E\right)-G^{R}\left(\mathbf{r}, \mathbf{r}^{\prime} ; E\right) \\
& \left.\times \Sigma^{<}\left(\mathbf{r}^{\prime}, \mathbf{r} ; E\right)-G^{<}\left(\mathbf{r}, \mathbf{r}^{\prime} ; E\right) \Sigma^{A}\left(\mathbf{r}^{\prime}, \mathbf{r} ; E\right)\right] .
\end{aligned}
$$

Depending on the nature of the interaction described by $\Sigma$, the scattering process may be highly nonlocal, in which case the above rate contains contributions from a large volume. The total interband current is found by integrating the divergence over the active volume and is equivalent to the total global transition rate and, via the Gauss theorem, to the difference of the interband currents at the boundaries of the interacting region. Making use of the cyclic property of the trace, it can be expressed in the form

$$
\begin{aligned}
R_{\mathrm{rad}}= & 2 \int d^{3} r \int_{B(\mathbf{r})} \frac{d E}{2 \pi \hbar} \int d^{3} r^{\prime}\left[\Sigma^{<}\left(\mathbf{r}, \mathbf{r}^{\prime} ; E\right) G^{>}\left(\mathbf{r}^{\prime}, \mathbf{r} ; E\right)\right. \\
& \left.-\Sigma^{>}\left(\mathbf{r}, \mathbf{r}^{\prime} ; E\right) G^{<}\left(\mathbf{r}^{\prime}, \mathbf{r} ; E\right)\right],
\end{aligned}
$$

with units $\left[R_{\mathrm{rad}}\right]=s^{-1}$. Within the general (basisindependent) NEGF picture, the right-hand side of Eq. (7) can be interpreted as follows: $\hbar^{-1} \Sigma^{<(>)}(E)$ represents the rate at which charge carriers with energy $E$ may leave (occupy) a state at that energy, whereas $G^{>(<)}(E)$ quantifies the energy resolved probability that the system can accept (donate) a particle of energy $E$, i.e., that there is an unoccupied (occupied) state at the right energy. The first term thus represents the total inscattering rate, while the second term provides the total outscattering rate.

If we are interested in the interband scattering rate, we can neglect in Eq. (7) the contributions to the self-energy from intraband scattering, e.g., via interaction with phonons, low-energy photons (free carrier absorption), or ionized impurities, since they cancel on energy integration over the band. However, if self-energies and Green's functions are determined self-consistently, as they should in order to guarantee current conservation, the Green's functions are related to the scattering self-energies via the Dyson equation for the propagator,

$$
\begin{aligned}
G^{R(A)}\left(\mathbf{r}_{1}, \mathbf{r}_{1^{\prime}} ; E\right)= & G_{0}^{R(A)}\left(\mathbf{r}_{1}, \mathbf{r}_{1^{\prime}} ; E\right) \\
& +\int d^{3} r_{2} \int d^{3} r_{3} G_{0}^{R(A)}\left(\mathbf{r}_{1}, \mathbf{r}_{2} ; E\right) \\
& \times \Sigma^{R(A)}\left(\mathbf{r}_{2}, \mathbf{r}_{3} ; E\right) G^{R(A)}\left(\mathbf{r}_{3}, \mathbf{r}_{1^{\prime}} ; E\right),
\end{aligned}
$$

and the Keldysh equation for the correlation functions,

$$
\begin{aligned}
G^{\lessgtr}\left(\mathbf{r}_{1}, \mathbf{r}_{1^{\prime}} ; E\right)= & \int d^{3} r_{2} \int d^{3} r_{3} G^{R}\left(\mathbf{r}_{1}, \mathbf{r}_{2} ; E\right) \\
& \times \Sigma^{\lessgtr}\left(\mathbf{r}_{2}, \mathbf{r}_{3} ; E\right) G^{A}\left(\mathbf{r}_{3}, \mathbf{r}_{1^{\prime}} ; E\right),
\end{aligned}
$$

and will thus be modified due to the intraband scattering. This means that in the case of self-consistent solutions, it is in general not possible to completely separate the effects of the different scattering processes, nor to isolate coherent from incoherent transport.
In the remainder of the paper, the general theory of interband transitions outlined above will be applied to optical interband transitions in direct and indirect semiconductors. For computational purposes and to ease comparison with existing descriptions, the theory will be reformulated using a simple effective mass band basis for completely homogeneous bulk systems and for inhomogeneous thin film devices.

\section{B. Bulk semiconductor}

For a homogeneous bulk system, the field operators for carriers in band $b$ can be written in the Bloch state basis,

$$
\hat{\Psi}_{b}(\mathbf{r}, t)=\sum_{\mathbf{k}} \psi_{b \mathbf{k}}(\mathbf{r}) \hat{c}_{b \mathbf{k}}(t) .
$$

The expression for the total radiative rate of carriers in band $b$ is simplified by using the Fourier space representation of Green's functions and self-energies $(O=G, \Sigma)$

$$
O_{b, b^{\prime}}\left(\mathbf{r}, \mathbf{r}^{\prime} ; E\right)=\sum_{\mathbf{k}} \psi_{b \mathbf{k}}(\mathbf{r}) O_{b, b^{\prime}}(\mathbf{k} ; E) \psi_{b^{\prime} \mathbf{k}}^{*}\left(\mathbf{r}^{\prime}\right),
$$

where $O_{b, b^{\prime}}(\mathbf{k} ; E)$ is the steady-state Fourier-transform of the real-time Keldysh components of the corresponding contourordered operator averages, e.g., the Green's function

$$
G_{b, b^{\prime}}\left(\mathbf{k} ; t-t^{\prime}\right)=\frac{1}{i \hbar}\left\langle\hat{T}_{\mathcal{C}}\left\{\hat{c}_{b \mathbf{k}}(t) \hat{c}_{b^{\prime} \mathbf{k}}^{\dagger}\left(t^{\prime}\right)\right\}\right\rangle .
$$

Inserting these expressions in (7), the band-resolved rates are obtained as

$$
\begin{aligned}
R_{\mathrm{rad}, b}= & 2 \int \frac{d E}{2 \pi \hbar} \sum_{\mathbf{k}} \sum_{b^{\prime}}\left[\Sigma_{b, b^{\prime}}^{<}(\mathbf{k} ; E) G_{b^{\prime}, b}^{>}(\mathbf{k} ; E)\right. \\
& \left.-\Sigma_{b, b^{\prime}}^{>}(\mathbf{k} ; E) G_{b^{\prime}, b}^{<}(\mathbf{k} ; E)\right] .
\end{aligned}
$$

In the following, the off-diagonal terms will be neglected $\left(O_{b} \equiv O_{b, b}\right)$, which means that only incoherent interband and subband polarization is considered.

\section{Direct interband transitions}

Inserting the electron-photon self-energy for a two-band model of a direct semiconductor (Appendix A) and neglecting intraband processes (free-carrier absorption and emission), the expression for the interband absorption rate becomes (in the following, the energy integration is restricted to the conduction band)

$$
\begin{aligned}
R_{\mathrm{abs}}= & 2 \int \frac{d E}{2 \pi \hbar} \sum_{\mathbf{k}} \sum_{\lambda, \mathbf{q}}\left|M_{c v}^{\gamma}(\mathbf{k}, \lambda, \mathbf{q})\right|^{2} N_{\lambda, \mathbf{q}}^{\gamma} \\
& \times G_{v}^{<}\left(\mathbf{k} ; E-\hbar \omega_{\lambda \mathbf{q}}\right) G_{c}^{>}(\mathbf{k} ; E),
\end{aligned}
$$

with $M_{c v}^{\gamma}$ the optical matrix element (Appendix A) and $N_{\lambda, \mathrm{q}}^{\gamma}$ the occupation of the photon modes. The latter is obtained from the modal photon flux via $N_{\lambda, \mathbf{q}}^{\gamma}=\phi_{\lambda, \mathbf{q}}^{\gamma} V / \tilde{c}$, where $\tilde{c}$ is the speed of the light in the active medium. The modal photon flux in turn is given by the modal intensity of the EM field as $\phi_{\lambda, \mathbf{q}}^{\gamma}=I_{\lambda, \mathbf{q}}^{\gamma} /\left(\hbar \omega_{\lambda \mathbf{q}}\right)$. Similarly, stimulated interband emission reads

$$
\begin{aligned}
R_{\mathrm{em}, \mathrm{st}}= & 2 \int \frac{d E}{2 \pi \hbar} \sum_{\mathbf{k}} \sum_{\lambda, \mathbf{q}}\left|M_{c v}^{\gamma}(\mathbf{k}, \lambda, \mathbf{q})\right|^{2} N_{\lambda, \mathbf{q}}^{\gamma} \\
& \times G_{v}^{>}\left(\mathbf{k} ; E-\hbar \omega_{\lambda \mathbf{q}}\right) G_{c}^{<}(\mathbf{k} ; E),
\end{aligned}
$$


while spontaneous interband emission is expressed as

$$
\begin{aligned}
R_{\mathrm{em}, \mathrm{sp}}= & 2 \int \frac{d E}{2 \pi \hbar} \sum_{\mathbf{k}} \sum_{\lambda, \mathbf{q}}\left|M_{c v}^{\gamma}(\mathbf{k}, \lambda, \mathbf{q})\right|^{2} \\
& \times G_{v}^{>}\left(\mathbf{k} ; E-\hbar \omega_{\lambda \mathbf{q}}\right) G_{c}^{<}(\mathbf{k} ; E) .
\end{aligned}
$$

The net absorption $R_{\mathrm{abs}, \text { net }}=R_{\mathrm{abs}}-R_{\mathrm{em}, \mathrm{st}}$ of photons in mode $(\lambda, \mathbf{q})$ is thus given by

$$
\begin{aligned}
R_{\text {abs,net }}(\lambda, \mathbf{q})= & 2 \int \frac{d E}{2 \pi \hbar} \sum_{\mathbf{k}}\left|M_{c v}^{\gamma}(\mathbf{k}, \lambda, \mathbf{q})\right|^{2} N_{\lambda, \mathbf{q}}^{\gamma} \\
& \times\left[G_{v}^{<}\left(\mathbf{k} ; E-\hbar \omega_{\lambda \mathbf{q}}\right) G_{c}^{>}(\mathbf{k} ; E)\right. \\
& \left.-G_{v}^{>}\left(\mathbf{k} ; E-\hbar \omega_{\lambda \mathbf{q}}\right) G_{c}^{<}(\mathbf{k} ; E)\right] .
\end{aligned}
$$

Conventionally, the rates for absorption and emission are calculated based on FGR, corresponding to the Born approximation within first-order perturbation theory. It is thus instructive to compare the above expressions with the FGR rate for net direct interband absorption, ${ }^{17}$

$$
\begin{aligned}
R_{\mathrm{abs}, \mathrm{net}}^{\mathrm{FGR}}(\lambda, \mathbf{q})= & 2 \sum_{\mathbf{k}} \frac{2 \pi}{\hbar}\left|M^{\gamma}(\mathbf{k}, \lambda, \mathbf{q})\right|^{2} N_{\lambda, \mathbf{q}}^{\gamma} \\
& \times \delta\left(\varepsilon_{c}(\mathbf{k})-\varepsilon_{v}(\mathbf{k})-\hbar \omega_{\lambda \mathbf{q}}\right)\left[f_{v}(\mathbf{k})-f_{c}(\mathbf{k})\right] .
\end{aligned}
$$

Here, the absorbing (bulk) material is described by the dispersion relations $\varepsilon_{b}(\mathbf{k}), b=c, v$, and assumed to be in a quasiequilibrium state with occupation described by the Fermi function $f_{b}(E)=\left(\exp \left[\beta\left(E-\mu_{b}\right)\right]+1\right)^{-1}, \beta=\left(k_{B} T\right)^{-1}$, where $\mu_{b}$, $b=c, v$ are global quasi-Fermi levels.

In order to reproduce the FGR result from the more general expression in terms of Green's functions, the unperturbed (i.e., noninteracting) equilibrium form of the latter needs to be used, which corresponds to the expressions for free fermions in equilibrium,

$$
\begin{gathered}
G_{b}^{<(0)}(\mathbf{k} ; E)=2 \pi i f_{b}(E) \delta\left(E-\varepsilon_{b}(\mathbf{k})\right), \\
G_{b}^{>(0)}(\mathbf{k} ; E)=2 \pi i\left[f_{b}(E)-1\right] \delta\left(E-\varepsilon_{b}(\mathbf{k})\right) .
\end{gathered}
$$

Introducing these expressions in (17) and carrying-out the energy integration reproduces the FGR expression (18).

To estimate the deviation of the rate from the FGR result for self-energies beyond the first Born approximation, the FGR rate is first used to derive the standard expression for the bulk absorption coefficient of the two-band effective mass model, which amounts to

$$
\alpha\left(\hbar \omega_{\gamma}\right)=\frac{R_{\mathrm{abs}, \mathrm{net}}\left(\hbar \omega_{\gamma}\right) / V}{S\left(\hbar \omega_{\gamma}\right) / \hbar \omega_{\gamma}},
$$

where $S\left(\hbar \omega_{\gamma}\right)$ is the monochromatic energy flux density of the EM field (i.e., the absolute value of the Poynting vector) given by

$$
S\left(\hbar \omega_{\gamma}\right)=\rho_{\gamma}\left(\hbar \omega_{\gamma}\right) \hbar \omega_{\gamma} \varepsilon_{b} \tilde{c} \int \frac{d \Omega}{4 \pi} \sum_{\lambda} N_{\lambda}^{\gamma}\left(\hbar \omega_{\gamma}, \Omega\right),
$$

where

$$
\rho_{\gamma}\left(\hbar \omega_{\gamma}\right)=\frac{\left(\hbar \omega_{\gamma}\right)^{2}}{2 \pi^{2}(\hbar \tilde{c})^{3}}
$$

is the photonic density of states of an optically isotropic medium with refractive index $n_{b}=\sqrt{\varepsilon_{b}}$ and corresponding speed of light $\tilde{c}=c_{0} / n$. With the standard approximation of isotropic and momentum-independent optical matrix elements, i.e., $\left|M_{c v}^{\gamma}(\mathbf{k}, \lambda, \mathbf{q})\right|^{2} \approx \overline{\mathcal{M}}_{c v}^{\gamma}\left(\hbar \omega_{\gamma}\right)$, the absorption rate is rewritten as follows:

$$
\begin{aligned}
R_{\mathrm{abs}}\left(\hbar \omega_{\gamma}\right) / V= & \overline{\mathcal{M}}_{c v}^{\gamma}\left(\hbar \omega_{\gamma}\right) \mathcal{J}_{c v}\left(\hbar \omega_{\gamma}\right) \rho_{\gamma}\left(\hbar \omega_{\gamma}\right) \\
& \times \int \frac{d \Omega}{4 \pi} \sum_{\lambda} N_{\lambda}^{\gamma}\left(\hbar \omega_{\gamma}, \Omega\right) \\
= & \overline{\mathcal{M}}_{c v}^{\gamma}\left(\hbar \omega_{\gamma}\right) \mathcal{J}_{c v}\left(\hbar \omega_{\gamma}\right) S\left(\hbar \omega_{\gamma}\right) /\left(\hbar \omega_{\gamma} \varepsilon_{b} \tilde{c}\right),
\end{aligned}
$$

which provides the bulk absorption coefficient

$$
\alpha\left(\hbar \omega_{\gamma}\right)=\tilde{\mathcal{M}}_{c v}^{\gamma}\left(\hbar \omega_{\gamma}\right) \mathcal{J}_{c v}\left(\hbar \omega_{\gamma}\right),
$$

with $\tilde{\mathcal{M}}_{c v}^{\gamma}=\overline{\mathcal{M}}_{c v}^{\gamma} /\left(\varepsilon_{b} \tilde{c}\right)$. The difference between FGR and NEGF approaches concerns the term $\mathcal{J}_{c v}\left(\hbar \omega_{\gamma}\right)$, which in the FGR case takes the specific form (using the continuum approximation $\left.\sum_{\mathbf{k}} \rightarrow \frac{V}{(2 \pi)^{3}} \int d^{3} k\right)$

$$
\begin{aligned}
\mathcal{J}_{c v}^{\mathrm{FGR}}\left(\hbar \omega_{\gamma}\right)= & \frac{2 \pi}{\hbar} \frac{2}{(2 \pi)^{3}} \int d^{3} k \delta\left[\varepsilon_{c}(\mathbf{k})-\varepsilon_{v}(\mathbf{k})-\hbar \omega_{\gamma}\right] \\
& \times\left[f_{v}(\mathbf{k})-f_{c}(\mathbf{k})\right] .
\end{aligned}
$$

If the occupation depends only marginally on crystalline momentum, the above expressions are related to the joint density of states $J_{c v}$ with suitable occupation,

$$
\begin{gathered}
\mathcal{J}_{c v}^{\mathrm{FGR}}\left(\hbar \omega_{\gamma}\right)=\frac{2 \pi}{\hbar} J_{c v}\left(\hbar \omega_{\gamma}\right)\left(f_{v}-f_{c}\right), \\
J_{c v}\left(\hbar \omega_{\gamma}\right)=\frac{2}{(2 \pi)^{3}} \int d^{3} k \delta\left[\varepsilon_{c}(\mathbf{k})-\varepsilon_{v}(\mathbf{k})-\hbar \omega_{\gamma}\right] .
\end{gathered}
$$

In the NEGF case, joint density of states and occupation cannot be separated but are both contained in the Keldysh Green's functions:

$$
\begin{aligned}
\mathcal{J}_{c v}^{\mathrm{GF}}\left(\hbar \omega_{\gamma}\right)= & \frac{2}{(2 \pi)^{3}} \int d^{3} k \int \frac{d E}{2 \pi \hbar}\left[G_{v}^{<}\left(\mathbf{k} ; E-\hbar \omega_{\gamma}\right) G_{c}^{>}(\mathbf{k} ; E)\right. \\
& \left.-G_{v}^{>}\left(\mathbf{k} ; E-\hbar \omega_{\gamma}\right) G_{c}^{<}(\mathbf{k} ; E)\right] \\
= & \hat{\mathcal{P}}_{c v, 0}\left(\mathbf{0}, \hbar \omega_{\gamma}\right),
\end{aligned}
$$

which via $\hat{\mathcal{P}}_{c v, 0}=\mathcal{P}_{c v, 0}^{>}-\mathcal{P}_{c v, 0}^{<}$is related to the Keldysh components of the free-carrier interband polarization function

$$
\begin{aligned}
\mathcal{P}_{c v, 0}^{\lessgtr}(\mathbf{q}, E)= & \frac{2}{(2 \pi)^{3}} \int d^{3} k \int \frac{d \tilde{E}}{2 \pi \hbar} G_{c}^{\lessgtr}(\mathbf{k} ; \tilde{E}) \\
& \times G_{v}^{\gtrless}(\mathbf{k}-\mathbf{q} ; \tilde{E}-E) .
\end{aligned}
$$

This expression is valid in any situation that can be described in terms of single-particle Green's functions. The deviations from the FGR result are marginal in the quasiequilibrium case and in absence of further interactions beyond electron-light coupling and of sources of nonequilibrium, which both modify the Green's functions, causing them to differ from the expressions given in Eqs. (19) and (20). It is straightforward to show that inserting the latter expressions in (30) reproduces (27), and for the special case of spherical bands, the joint density of states is given by the well-known analytical expression

$$
J_{c v}^{\operatorname{dir}}\left(\hbar \omega_{\gamma}\right)=\frac{\left(2 m_{r}^{*}\right)^{\frac{3}{2}}}{\pi^{2} \hbar^{3}} \sqrt{E_{\gamma}-E_{g}}
$$

where $m_{r}^{*}$ is the reduced effective mass and $E_{g}$ is the direct band gap. 


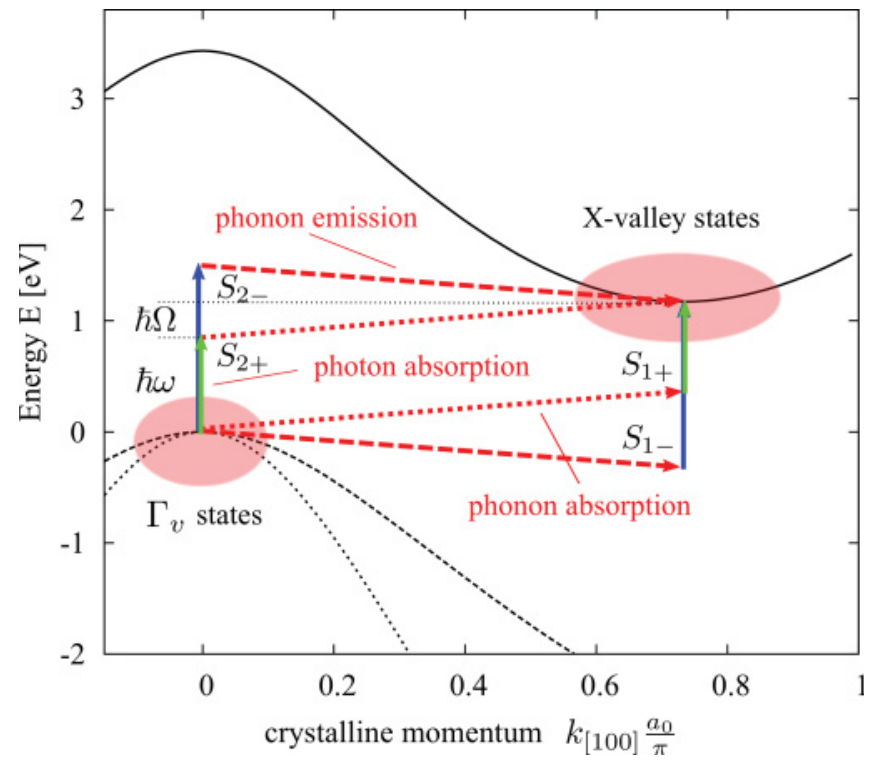

FIG. 1. (Color online) Possible excitation pathways for optical interband transitions in indirect semiconductors on the example of bulk silicon.

\section{Phonon-assisted interband transitions}

Even in the case of a direct semiconductor discussed above, the presence of phonons can have a considerable effect on the optical transition rates as new excitation paths become available, i.e., the phonons strongly increase the number of initial-final state pairs separated by a given transition energy. However, comparing to the direct transition, this enhancement of the joint density of states is overcompensated by the fact that the transitions become much more unlikely due to the need for coupling to a suitable phonon and the detour via the virtual state. As a consequence, phonon-assisted transitions may be neglected in direct bulk semiconductors where crystalline momentum is conserved. Obviously, the situation differs completely in indirect bulk semiconductors, where no direct transitions are possible. There, at lowest nonvanishing order, four different excitation processes exist (Fig. 1):

$S_{1+}$ : 1. phonon absorption, 2. photon absorption,

$S_{1-}$ : 1. phonon emission, 2. photon absorption,

$S_{2+}$ : 1. photon absorption, 2. phonon absorption,

$S_{2-}: 1$. photon absorption, 2. phonon emission.

To exemplify the inclusion of the phonon scattering in the NEGF description of optical interband transitions, we will in
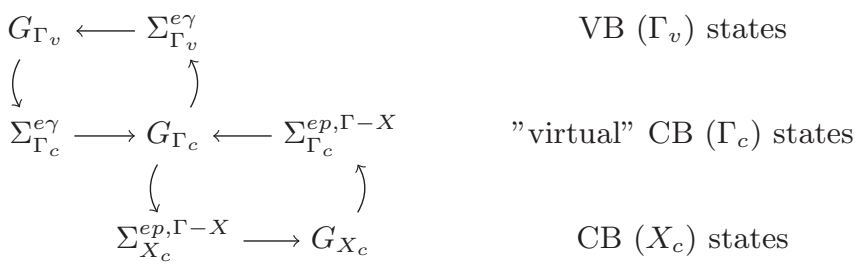

FIG. 2. Self-consistent computation of Green's functions and scattering self-energies in silicon enabling the description of phononassisted indirect optical transitions

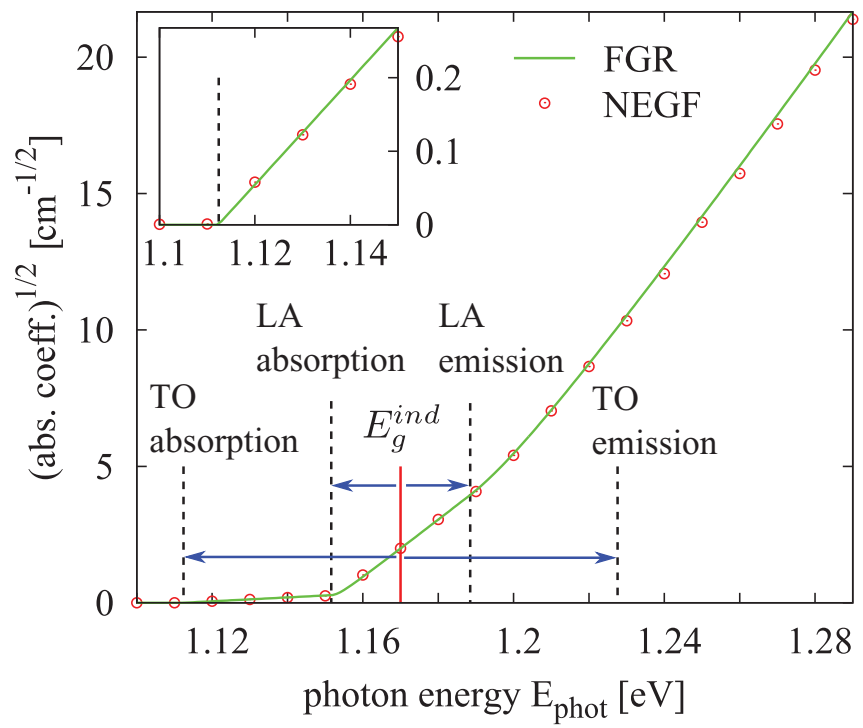

FIG. 3. (Color online) Photon absorption coefficient for indirect optical transitions in intrinsic bulk silicon: FGR (full line) and NEGF (symbols) rates are in close agreement, since the correction fo the conduction band DOS due to the phonon-mediated $\Gamma-X$ scattering is weak. The arrows indicate the effect of the electronic interaction with specific phonon modes on the absorption characteristics, with the length of the arrows corresponding to the energy of the associated phonon mode.

the following focus on processes $S_{2 \pm}$. The FGR transition rate for these processes are obtained from second-order perturbation theory as

$$
\begin{aligned}
R_{\mathrm{abs}, \pm}^{\mathrm{FGR}}(\lambda, \mathbf{q})= & \frac{2 \pi}{\hbar} N_{\lambda, \mathbf{q}}^{\gamma} \sum_{\mathbf{k}_{c}, \mathbf{k}_{v}} \sum_{\Lambda, \mathbf{Q}} \frac{\left[M_{\Lambda, \mathbf{Q}}^{\mathrm{ph}}\left(\mathbf{k}_{v}, \mathbf{k}_{c}\right)\right]^{2}\left[M_{\lambda, \mathbf{q}}^{\gamma}\left(\mathbf{k}_{v}\right)\right]^{2}}{\left|\varepsilon_{c}\left(\mathbf{k}_{v}\right)-\varepsilon_{v}\left(\mathbf{k}_{v}\right)-\hbar \omega_{\lambda \mathbf{q}}\right|^{2}} \\
& \times\left[N_{\Lambda, \mathbf{Q}}^{\mathrm{ph}}+\frac{1}{2} \mp \frac{1}{2}\right] f_{v}\left(\mathbf{k}_{v}\right)\left[1-f_{c}\left(\mathbf{k}_{c}\right)\right] \\
& \times \delta\left(\varepsilon_{c}\left(\mathbf{k}_{c}\right)-\varepsilon_{v}\left(\mathbf{k}_{v}\right)-\hbar \omega_{\lambda \mathbf{q}} \mp \hbar \Omega_{\Lambda, \mathbf{Q}}\right) .
\end{aligned}
$$

Here, $M_{\Lambda, Q}^{\mathrm{ph}}$ encodes the matrix element for coupling of electrons to the phonon mode $(\Lambda, \mathbf{Q})$ with energy $\hbar \Omega_{\Lambda, \mathbf{Q}}$ and occupation given by the Bose-Einstein distribution $N_{\Lambda, \mathbf{Q}}^{\mathrm{ph}}=$ $\left(e^{\beta \hbar \Omega_{\Lambda, Q}}-1\right)^{-1}, \beta=\left(k_{B} T\right)^{-1}$ at lattice temperature $T$. In analogy to the direct case, with the previously used approximations for the optical matrix elements and the additional assumptions

$$
\begin{gathered}
\left|\varepsilon_{c}\left(\mathbf{k}_{v}\right)-\varepsilon_{v}\left(\mathbf{k}_{v}\right)-\hbar \omega_{\lambda \mathbf{q}}\right| \approx\left|E_{g 0}-\hbar \omega_{\lambda \mathbf{q}}\right| \\
M_{\Lambda, \mathbf{Q}}^{\mathrm{ph}}\left(\mathbf{k}_{v}, \mathbf{k}_{c}\right) \approx \mathcal{M}_{\Lambda}^{\mathrm{ph}} \delta\left(\mathbf{k}_{v}-\mathbf{k}_{c}+\mathbf{Q}\right),
\end{gathered}
$$

we can write the (phonon-assisted) absorption coefficient

$$
\begin{aligned}
\alpha^{\mathrm{ind}}\left(\hbar \omega_{\gamma}\right)= & \frac{\tilde{\mathcal{M}}_{c v}^{\gamma}\left(\hbar \omega_{\gamma}\right)^{2}}{\left|E_{g 0}-\hbar \omega_{\gamma}\right|^{2}} \sum_{\Lambda} \mathcal{M}_{\Lambda}^{\mathrm{ph}} \\
& \times \sum_{s= \pm}\left[N_{\Lambda}^{\mathrm{ph}}+\frac{1}{2}-s \frac{1}{2}\right] \mathcal{J}_{c v, s}^{\text {ind }}\left(\hbar \omega_{\gamma}\right),
\end{aligned}
$$


but where now the joint density of states for indirect transitions is used in (28),

$$
\begin{aligned}
J_{c v, \pm}^{\text {ind }}\left(\hbar \omega_{\gamma}\right)= & \frac{2}{(2 \pi)^{6}} \int d^{3} k_{1} \int d^{3} k_{2} \delta\left[\varepsilon_{c}\left(\mathbf{k}_{1}\right)-\varepsilon_{v}\left(\mathbf{k}_{2}\right)\right. \\
& \left.-\hbar \omega_{\gamma} \mp \hbar \Omega_{\Lambda, \mathbf{k}_{1}-\mathbf{k}_{2}}\right],
\end{aligned}
$$

which for spherical bands and a single phonon frequency $\Omega$ may be simplified to

$$
J_{c v, \pm}^{\text {ind }}\left(\hbar \omega_{\gamma}\right)=\frac{\left(m_{c}^{*} m_{v}^{*}\right)^{\frac{3}{2}}}{(2 \pi \hbar)^{3}}\left(\hbar \omega_{\gamma}-E_{g} \mp \hbar \Omega\right)^{2},
$$

where $E_{g}$ denotes the (indirect) band gap.

In the following, the phonon-assisted absorption rate shall be derived within the NEGF formalism, starting from the expression for the absorption rate, but where now the Green's functions contain the contributions due to the electron-phonon scattering. Since the Green's functions and interaction selfenergies are evaluated in a self-consistency iteration process, all possible single phonon processes are included to all orders, i.e., the Green's functions contain contributions from a number of scattering processes that corresponds to the number of self-consistency iteration steps. It is thus via this self-consistent computation that phonon-assisted optical transitions are enabled, even though the self-energies themselves are only on the level of a first order self-consistent Born approximation, i.e., do not include the combination of both electron-phonon and electron-photon scattering at the same time. In Fig. 2, this procedure is shown for the technologically relevant example of indirect interband absorption of photons in silicon, where zone-boundary phonons provide the wave-vector difference in a $\Gamma-X$ intervalley scattering process.

We start again from the general expression for the inscattering rate, which in this case reads

$$
R_{\text {in }}=2 \int \frac{d E}{2 \pi \hbar} \sum_{\mathbf{k}} \Sigma_{X_{c}}^{<}(\mathbf{k} ; E) G_{X_{c}}^{>}(\mathbf{k} ; E) .
$$

Assuming a photon-first indirect process $\left(S_{2 \pm}\right)$, the corresponding expression for $\Gamma-X$ scattering is inserted for the self-energy [see Eq. (A28) in Appendix A], leading to the equivalent of Eq. (14) ( $\sigma=\mathrm{LA}, \mathrm{TO}$ : phonon mode),

$$
\begin{aligned}
R_{\text {in }}= & 2 \int \frac{d E}{2 \pi \hbar} \sum_{\mathbf{k}} \sum_{\mathbf{Q}, \sigma}\left[M_{\sigma}^{\mathrm{ph}}\left(\Omega_{\sigma}\right)\right]^{2}\left[N_{\sigma}^{\mathrm{ph}} G_{\Gamma_{c}}^{<}\left(\mathbf{Q} ; E-\hbar \Omega_{\sigma}\right)\right. \\
& \left.+\left(N_{\sigma}^{\mathrm{ph}}+1\right) G_{\Gamma_{c}}^{<}\left(\mathbf{Q} ; E+\hbar \Omega_{\sigma}\right)\right] G_{X_{c}}^{>}(\mathbf{k} ; E) .
\end{aligned}
$$

In the next step, the Keldysh equation for electron-photon interaction is used to replace the lesser $\Gamma_{c}-\mathrm{GF}$,

$$
G_{\Gamma_{c}}^{<}(\mathbf{k} ; E)=G_{\Gamma_{c}}^{R}(\mathbf{k} ; E) \Sigma_{\Gamma_{c}}^{<\gamma}(\mathbf{k} ; E) G_{\Gamma_{c}}^{A}(\mathbf{k} ; E),
$$

providing the modal absorption rate

$$
\begin{aligned}
R_{\mathrm{abs}}(\lambda, \mathbf{q})= & 2 \int \frac{d E}{2 \pi \hbar} \sum_{\mathbf{k}} \sum_{\mathbf{Q}, \sigma}\left[M_{\sigma}^{\mathrm{ph}}\left(\Omega_{\sigma}\right)\right]^{2}\left[M^{\gamma}(\mathbf{k}, \lambda, \mathbf{q})\right]^{2} \\
& \times N_{\lambda, \mathbf{q}}^{\gamma}\left[N_{\sigma}^{\mathrm{ph}} G_{\Gamma_{c}}^{R}\left(\mathbf{Q} ; E-\hbar \Omega_{\sigma}\right)\right. \\
& \times G_{\Gamma_{v}}^{<}\left(\mathbf{Q} ; E-\hbar \Omega_{\sigma}-\hbar \omega_{\lambda \mathbf{q}}\right) \\
& \times G_{\Gamma_{c}}^{A}\left(\mathbf{Q} ; E-\hbar \Omega_{\sigma}\right)+\left(N_{\sigma}^{\mathrm{ph}}+1\right) \\
& \times G_{\Gamma_{c}}^{R}\left(\mathbf{Q} ; E+\hbar \Omega_{\sigma}\right) \\
& \left.\times G_{\Gamma_{v}}^{<}\left(\mathbf{Q} ; E+\hbar \Omega_{\sigma}-\hbar \omega_{\lambda \mathbf{q}}\right) G_{\Gamma_{c}}^{A}\left(\mathbf{Q} ; E+\hbar \Omega_{\sigma}\right)\right] \\
& \times G_{X_{c}}^{>}(\mathbf{k} ; E) .
\end{aligned}
$$

Again, this is to be compared to the FGR result, which, for the same electron-photon and electron-phonon interaction Hamiltonian terms, follows from second-order perturbation theory to

$$
\begin{aligned}
R_{\mathrm{abs}}^{\mathrm{FGR}}(\lambda, \mathbf{q})= & \frac{2 \pi}{\hbar} \sum_{\mathbf{k}_{c}, \mathbf{k}_{v}} \sum_{\sigma} \frac{\left[M_{\sigma}^{\mathrm{ph}}\left(\Omega_{\sigma}\right)\right]^{2}\left[M^{\gamma}\left(\mathbf{k}_{v}, \lambda, \mathbf{q}\right)\right]^{2} N_{\lambda, \mathbf{q}}^{\gamma}}{\left|\varepsilon_{\Gamma_{c}}\left(\mathbf{k}_{v}\right)-\varepsilon_{\Gamma_{v}}\left(\mathbf{k}_{v}\right)-\hbar \omega_{\lambda \mathbf{q}}\right|^{2}} \\
& \times\left\{N_{\sigma}^{\mathrm{ph}} \delta\left[\varepsilon_{X_{c}}\left(\mathbf{k}_{c}\right)-\varepsilon_{\Gamma_{v}}\left(\mathbf{k}_{v}\right)-\hbar \omega_{\lambda \mathbf{q}}-\hbar \Omega_{\sigma}\right]\right. \\
& +\left(N_{\sigma}^{\mathrm{ph}}+1\right) \delta\left[\varepsilon_{X_{c}}\left(\mathbf{k}_{c}\right)-\varepsilon_{\Gamma_{v}}\left(\mathbf{k}_{v}\right)-\hbar \omega_{\lambda \mathbf{q}}\right. \\
& \left.\left.+\hbar \Omega_{\sigma}\right]\right\} f_{\Gamma_{v}}\left(\mathbf{k}_{v}\right)\left[1-f_{X_{c}}\left(\mathbf{k}_{c}\right)\right] .
\end{aligned}
$$

Now, inserting the noninteracting equilibrium expressions for the lesser, greater, and retarded GF in (43) provides the expression

$$
\begin{aligned}
R_{\mathrm{abs}}(\lambda, \mathbf{q})= & 2 \int \frac{d E}{2 \pi \hbar} \sum_{\mathbf{k}} \sum_{\mathbf{Q}, \sigma}\left[M_{\sigma}^{\mathrm{ph}}\left(\Omega_{\sigma}\right)\right]^{2}\left[M^{\gamma}(\mathbf{k}, \lambda, \mathbf{q})\right]^{2} N_{\lambda, \mathbf{q}}^{\gamma}\left\{\frac{N_{\sigma}^{\mathrm{ph}} i f_{\Gamma_{v}}\left(E-\hbar \Omega_{\sigma}-\hbar \omega_{\lambda \mathbf{q}}\right) 2 \pi \delta\left(E-\hbar \Omega_{\sigma}-\hbar \omega_{\lambda \mathbf{q}}-\varepsilon_{\Gamma_{v}}(\mathbf{Q})\right)}{\left|E-\hbar \Omega_{\sigma}-\hbar \omega_{\lambda \mathbf{q}}-\varepsilon_{\Gamma_{c}}(\mathbf{Q})+i \eta_{0+}\right|^{2}}\right. \\
& \left.+\frac{\left(N_{\sigma}^{\mathrm{ph}}+1\right) i f_{\Gamma_{v}}\left(E+\hbar \Omega_{\sigma}-\hbar \omega_{\lambda \mathbf{q}}\right) 2 \pi \delta\left[E+\hbar \Omega_{\sigma}-\hbar \omega_{\lambda \mathbf{q}}-\varepsilon_{\Gamma_{v}}(\mathbf{Q})\right]}{\left|E+\hbar \Omega_{\sigma}-\hbar \omega_{\lambda \mathbf{q}}-\varepsilon_{\Gamma_{c}}(\mathbf{Q})+i \eta_{0+}\right|^{2}}\right\} i\left[f_{X_{c}}(E)-1\right] 2 \pi \delta\left(E-\varepsilon_{X_{c}}(\mathbf{k})\right),
\end{aligned}
$$

which, on performing the energy integration and with $\mathbf{Q}=\mathbf{k}_{v}$, $\mathbf{k}=\mathbf{k}_{c}$, reproduces again the FGR result given in (44).

For the numerical implementation, a simple three-band effective mass model for the electronic structure of silicon is used. The $X$ electrons are described by a multivalley picture with identical values for transverse and longitudinal effective mass, and the $\Gamma_{v}$ holes as well as the virtual $\Gamma_{c}$ states used in the indirect transitions are modelled by single effective mass bands. The holes are modelled by a single effective mass corresponding to heavy holes. The band structure and interaction parameters used in the numerical examples are listed in Table I. Figure 3 displays the close agreement between NEGF and FGR for equilibrium bulk absorption close to the indirect band edge and the characteristic fingerprints of the involved TO and LA phonon modes.

\section{Thin film devices}

In the case of ultra-thin-film and especially heteromultilayer devices, translational invariance does no longer apply in 
TABLE I. Material parameters used in simulations.

\begin{tabular}{lccccc}
\hline \hline$m_{\Gamma c}^{*}$ & $m_{X}^{*}$ & $m_{\Gamma v}^{*}$ & $E_{g, \Gamma v-\Gamma c}$ & $E_{g, \Gamma v-X}$ & $P_{c v}^{2} / m_{0}$ \\
\hline $0.3 m_{0}$ & $0.3 m_{0}$ & $0.54 m_{0}$ & $3.1 \mathrm{eV}$ & $1.17 \mathrm{eV}$ & $4 \mathrm{eV}$ \\
$\sigma$ & Mode & $\hbar \Omega_{\sigma}$ & $D_{i v} K_{\sigma}$ & & \\
\hline$(\Gamma-\mathrm{X})_{1}$ & LA & $18.4 \mathrm{meV}$ & $2.45 \times 10^{8} \mathrm{eV} / \mathrm{cm}$ & & \\
$(\Gamma-\mathrm{X})_{2}$ & TO & $57.6 \mathrm{meV}$ & $0.8 \times 10^{8} \mathrm{eV} / \mathrm{cm}$ & & \\
\hline \hline
\end{tabular}

growth direction. For such a system, the appropriate representation of the field operators has the form

$$
\hat{\Psi}_{b}(\mathbf{r}, t)=\sum_{\mathbf{k}_{\|}, i} \psi_{i b \mathbf{k}_{\|}}(\mathbf{r}) \hat{c}_{i b \mathbf{k}_{\|}}(t)
$$

with the basis functions

$$
\psi_{i n \mathbf{k}_{\|}}(\mathbf{r})=\varphi_{i \mathbf{k}_{\|}}(\mathbf{r}) u_{n \mathbf{k}_{0}}(\mathbf{r})
$$

where $\varphi_{i \mathbf{k}_{\|}}$is the envelope basis function for discrete spatial (layer) index $i$ (longitudinal) and transverse momentum $\mathbf{k}_{\|}$, $u_{n \mathbf{k}_{0}}$ is the Bloch function of bulk band $n$, centered on $\mathbf{k}_{0}$. In the case of a system with large transverse extension, the envelope basis function can be written as

$$
\varphi_{i \mathbf{k}_{\|}}(\mathbf{r})=\frac{e^{i \mathbf{k}_{\|} \mathbf{r}_{\|}}}{\sqrt{\mathcal{A}}} \chi_{i}(z)
$$

where $\mathbf{r}_{\|}=(x, y), \mathcal{A}$ is the cross-sectional area, and $\chi_{i}$ is the longitudinal envelope basis function. For the latter, finite element shape functions are a popular choice. ${ }^{11,18}$ Here, we will use a simple finite difference basis equivalent to a separate single band tight-binding approach for each band, ${ }^{19-21}$

$$
\chi_{i}(z)=\left[\theta\left(z-z_{i}\right)-\theta\left(z-z_{i+1}\right)\right] / \sqrt{\Delta},
$$

where $\Delta$ is the spatial discretization step. In the above basis, the Green's functions and self-energies have the potentially nonlocal representation $(O=G, \Sigma)$

$$
O_{b, b^{\prime}}\left(\mathbf{r}, \mathbf{r}^{\prime} ; E\right)=\sum_{\mathbf{k}_{\|}} \sum_{i, j} \psi_{i b \mathbf{k}_{\|}}(\mathbf{r}) O_{i b, j b^{\prime}}\left(\mathbf{k}_{\|} ; E\right) \psi_{j b^{\prime} \mathbf{k}_{\|}}^{*}\left(\mathbf{r}^{\prime}\right),
$$

where the contour-ordered steady-state Green's functions are now defined as

$$
G_{i b, j b^{\prime}}\left(\mathbf{k}_{\|} ; t-t^{\prime}\right)=\frac{1}{i \hbar}\left\langle\hat{T}_{\mathcal{C}}\left\{\hat{c}_{i b \mathbf{k}_{\|}}(t) \hat{c}_{j b^{\prime} \mathbf{k}_{\|}}^{\dagger}\left(t^{\prime}\right)\right\}\right\rangle .
$$

The NEGF formalism employed here for the thin film device follows the approach taken in Ref. 22, but with the simple band structure model introduced in Sec. II B. The device under consideration consists basically of a slab of undoped absorber material sandwiched between thin doped layers, which make ohmic contacts to extended flat-band bulk contact regions with fixed chemical potential. The Green's functions for the charge carriers are determined by solving the linear system corresponding to Eq. (8) and (9) in the local basis (47),

$$
\begin{gathered}
\mathbf{G}^{R}(v)=\left[\left\{\mathbf{G}_{0}^{R}(v)\right\}^{-1}-\mathbf{\Sigma}^{R I}(v)-\mathbf{\Sigma}^{R B}(v)\right]^{-1}, \\
\mathbf{G}_{0}^{R}(v)=\left[(E+i \eta) 11-\mathbf{H}^{(0)}\left(\mathbf{k}_{\|}\right)\right]^{-1}, \\
\mathbf{G}^{A}(v)=\left[\mathbf{G}^{R}(v)\right]^{\dagger}, \\
\mathbf{G}^{\lessgtr}(v)=\mathbf{G}^{R}(v)\left[\boldsymbol{\Sigma}^{\lessgtr I}(v)+\mathbf{\Sigma}^{\lessgtr B}(v)\right] \mathbf{G}^{A}(v),
\end{gathered}
$$

where $v=\left(\mathbf{k}_{\|} ; E\right)$. The noninteracting Hamiltonian $\mathbf{H}^{(0)}$ contains, in addition to the electronic structure of noninteracting carriers, the Hartree potential of Coulomb interaction, corresponding to the mean-field treatment of the carrier-carrier interaction, which is obtained from the solution of Poisson's equation for the given profile of ionized dopants and the carrier density obtained from the carrier Green's functions, e.g., in the case of the conduction electrons

$$
n_{i}=\sum_{b=\Gamma_{c}, X} f_{b} \sum_{\mathbf{k}_{\|}} \int \frac{d E}{\pi \mathcal{A} \Delta}(-i) G_{i i, b}^{<}\left(\mathbf{k}_{\|} ; E\right),
$$

where $f_{b}$ denotes the valley degeneracy of the conduction bands, which is $f_{\Gamma_{c}}=1$ and $f_{X}=6$. A similar expression is found for the hole density. In the solution of Poisson's equation, open boundary conditions are imposed, corresponding to the requirement of global charge neutrality. Due to the interdependence of the NEGF and Poisson equations via charge density and Hartree potential, they need to be solved in an additional self-consistency iteration process. The terms $\Sigma^{\cdot B}$ denote the components of the contact self-energy, which in this case is obtained by electronic mode-matching to the bulk Bloch states of the flat-band contact region. ${ }^{23}$ The boundary-self energy enables the explicit description of carrier injection and extraction of carriers at contacts and corresponds to the imposition of open boundary conditions for the carrier wave functions. The components $\Sigma^{\cdot I}$ are due to the interactions of electrons with photons and phonons. The expressions for these interaction self-energies are determined following the same approach as in the bulk case, i.e., as the Fock term within many-body perturbation theory on the level of a self-consistent Born approximation, and are discussed

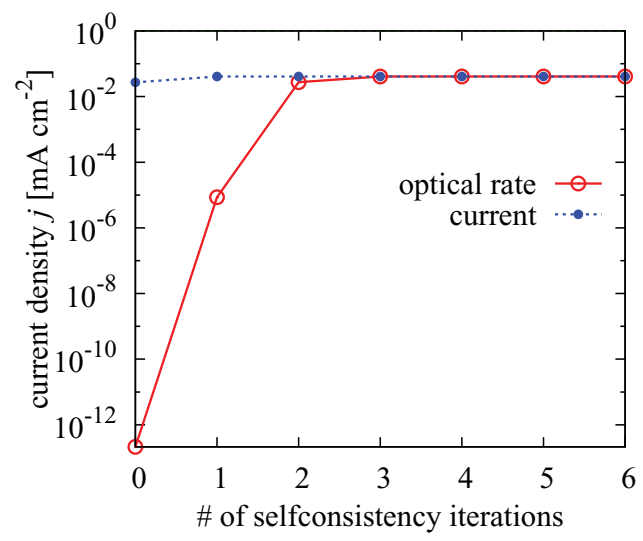

FIG. 4. (Color online) Convergence of the interband photogeneration rate (integrated over growth dimension) and of the resulting total interband current density in the NEGF self-consistency iteration process (Fig. 2). The device under consideration is a 73-nm-thick silicon $p-i-n$ diode with contact regions formed by $13.5-\mathrm{nm}$ doped layers of low doping level $\left(10^{16} \mathrm{~cm}^{-3}\right)$. 


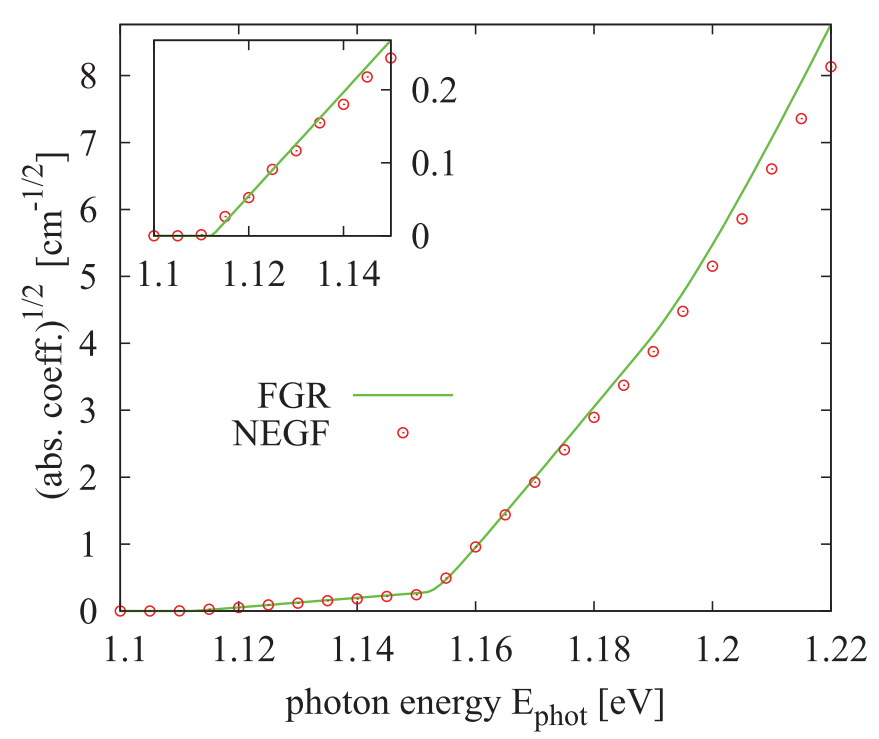

FIG. 5. (Color online) Photon absorption coefficient for indirect optical transitions, computed with the thin film NEGF model (dots) for the 73-nm-thick quasi-intrinsic silicon $p-i-n$ diode, in comparison with the absorption coefficient obtained from the Fermi golden rule rate for bulk silicon (full line).

in the Appendix. Since the Green's function elements off-diagonal in the band index are neglected, band coupling appears only via the self-energies of the scattering processes.

For thin films, the conservation law corresponding to Eq. (4) for the divergence of the electron current between model layers $i-1$ and $i$ becomes $\left[v=\left(\mathbf{k}_{\|} ; E\right)\right]$

$$
\begin{aligned}
\frac{J_{i}-J_{i-1}}{\Delta}= & -\frac{2}{\hbar \Delta} \sum_{\mathbf{k}_{\|}} \int \frac{d E}{2 \pi}\left[\boldsymbol{\Sigma}^{R}(v) \mathbf{G}^{<}(v)-\mathbf{G}^{R}(v) \boldsymbol{\Sigma}^{<}(v)\right. \\
& \left.+\boldsymbol{\Sigma}^{<}(v) \mathbf{G}^{A}(v)-\mathbf{G}^{<}(v) \boldsymbol{\Sigma}^{A}(v)\right]_{i, i} \\
\equiv & R_{\mathrm{rad}}\left(z_{i}\right),
\end{aligned}
$$

where $R_{\mathrm{rad}}(z)$ is again the local radiative rate. Making use of the cyclic property of the trace, the total radiative rate

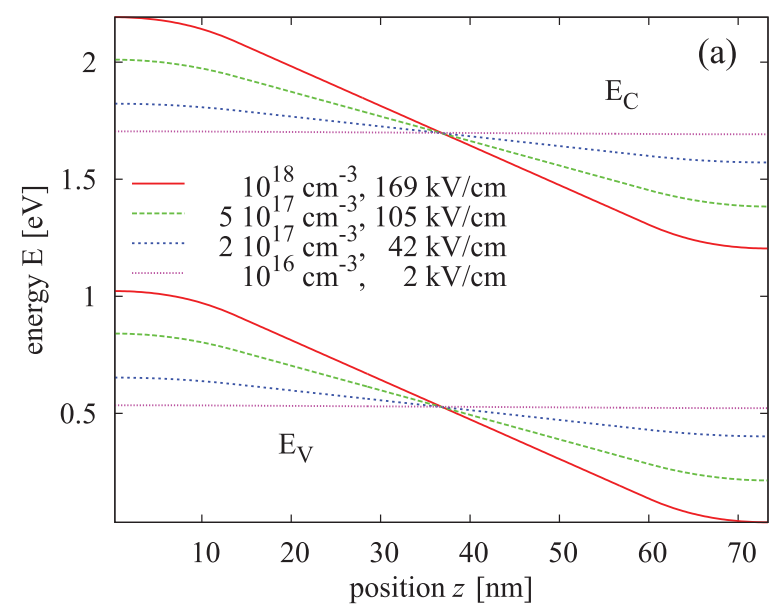

follows as

$$
\begin{aligned}
R_{\mathrm{rad}}= & \frac{2}{\hbar} \int_{B} \frac{d E}{2 \pi} \operatorname{Tr}\left\{\sum _ { \mathbf { k } _ { \| } } \left[\mathbf{\Sigma}_{e \gamma}^{<}\left(\mathbf{k}_{\|} ; E\right) \mathbf{G}^{>}\left(\mathbf{k}_{\|} ; E\right)\right.\right. \\
& \left.\left.-\boldsymbol{\Sigma}_{e \gamma}^{>}\left(\mathbf{k}_{\|} ; E\right) \mathbf{G}^{<}\left(\mathbf{k}_{\|} ; E\right)\right]\right\} \\
\equiv & \Delta \sum_{i} R_{\mathrm{rad}}\left(z_{i}\right)=\sum_{i}\left(J_{i}^{B}-J_{i-1}^{B}\right) \\
\equiv & J_{N}^{B}-J_{1}^{B} \equiv \begin{cases}J_{N}, & B=C B \\
J_{1}, & B=V B .\end{cases}
\end{aligned}
$$

In the case of the indirect gap material considered here, the electronic current density as given by the Green's function and the nearest-neighbor interlayer hopping matrix $\mathbf{t}$ is

$$
\begin{aligned}
j_{i}= & \sum_{b=\Gamma_{c}, X} f_{b} \sum_{\mathbf{k}_{\|}} \int \frac{d E}{\pi \hbar \mathcal{A}}\left[t_{i i+1} G_{i+1 i, b}^{<}\left(\mathbf{k}_{\|} ; E\right)\right. \\
& \left.-t_{i+1 i} G_{i i+1, b}^{<}\left(\mathbf{k}_{\|} ; E\right)\right],
\end{aligned}
$$

the total radiative rate is

$$
\begin{aligned}
R_{e \gamma}= & \frac{2}{\hbar} \int_{\Gamma_{c}} \frac{d E}{2 \pi} \operatorname{Tr}\left\{\sum _ { \mathbf { k } _ { \| } } \left[\boldsymbol{\Sigma}_{e \gamma, \Gamma_{c}}^{<}\left(\mathbf{k}_{\|} ; E\right) \mathbf{G}_{\Gamma_{c}}^{>}\left(\mathbf{k}_{\|} ; E\right)\right.\right. \\
& \left.\left.-\boldsymbol{\Sigma}_{e \gamma, \Gamma_{c}}^{>}\left(\mathbf{k}_{\|} ; E\right) \mathbf{G}_{\Gamma_{c}}^{<}\left(\mathbf{k}_{\|} ; E\right)\right]\right\},
\end{aligned}
$$

and the intervalley phonon scattering rate reads

$$
\begin{aligned}
R_{\mathrm{ep}, \Gamma-\mathrm{X}}= & \frac{2}{\hbar} \int_{\Gamma_{c}} \frac{d E}{2 \pi} \operatorname{Tr}\left\{\sum _ { \mathbf { k } _ { \| } } \left[\boldsymbol{\Sigma}_{\mathrm{ep}(\Gamma-\mathrm{X}), \Gamma_{c}}^{<}\left(\mathbf{k}_{\|} ; E\right)\right.\right. \\
& \left.\left.\times \mathbf{G}_{\Gamma_{c}}^{>}\left(\mathbf{k}_{\|} ; E\right)-\boldsymbol{\Sigma}_{\mathrm{ep}(\Gamma-\mathrm{X}), \Gamma_{c}}^{>}\left(\mathbf{k}_{\|} ; E\right) \mathbf{G}_{\Gamma_{c}}^{<}\left(\mathbf{k}_{\|} ; E\right)\right]\right\} .
\end{aligned}
$$

The convergence of scattering rate and interband current as a function of self-consistency iteration steps is shown in Fig. 4 for a $p-i-n$ diode of $73 \mathrm{~nm}$ total thickness, with a central undoped region of $46 \mathrm{~nm}$ width sandwiched between thin doped layers of $13.5 \mathrm{~nm}$ thickness each and low doping levels

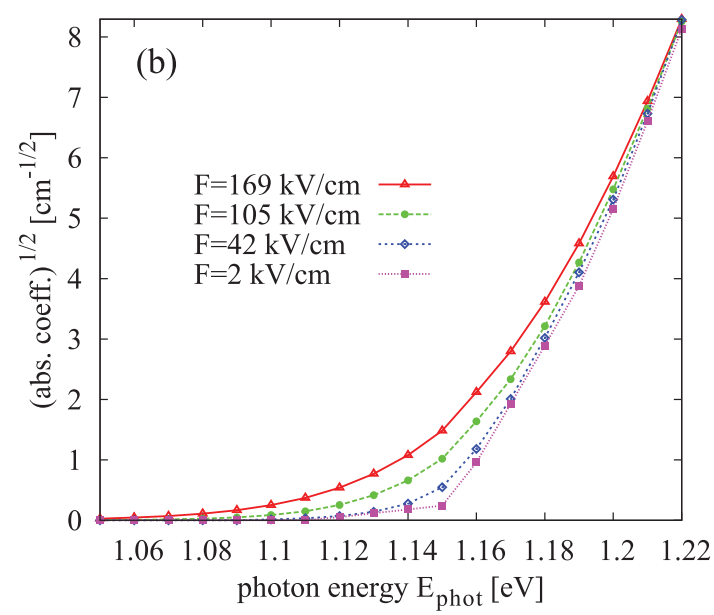

FIG. 6. (Color online) (a) Band profile of the 73-nm-thick silicon $p-i-n$ junction device from the self-consistent solution of the NEGFPoisson equations for indicated doping levels ranging from $N_{d}=10^{16} \mathrm{~cm}^{-3}$ to $N_{d}=10^{18} \mathrm{~cm}^{-3}$. The corresponding fields in the undoped region vary from $F=2 \mathrm{kV} / \mathrm{cm}$ to $F=169 \mathrm{kV} / \mathrm{cm}$. The equilibrium Fermi level lies at $1.1 \mathrm{eV}$. (b) Near band gap optical absorption rate in the intrinsic region of a silicon $p-i-n$ junction for various values of the built-in field corresponding to different doping levels. 


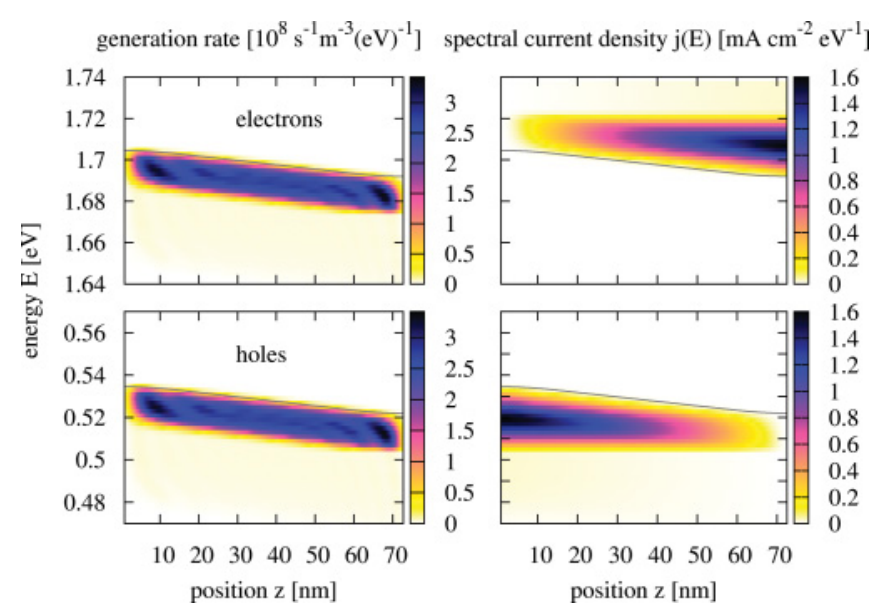

FIG. 7. (Color online) Energy resolved local generation rate in the virtual $\Gamma_{c}$ state and resulting local photocurrent spectrum in the $X$-valley band at very low internal field $F=2 \mathrm{kV} / \mathrm{cm}$. The gray lines indicate the band edges ( $X$ valley for electrons).

of $10^{16} \mathrm{~cm}^{-3}$. The convergence is fast due to the low carrier density in the absorbing quasi-intrinsic

Figure 5 shows the indirect optical absorption for the quasiintrinsic slab of the indirect gap semiconductor discussed above. In this case of near flat-band conditions, the bulk absorption is retrieved close to the band edge. For the more interesting case of an ultra-thin bipolar junction with high doping, the global absorption coefficient starts to deviate from the bulk value due to the strong internal field. This so-called Franz-Keldysh effect is displayed in Fig. 6.

Figure 7 shows the energy resolved local generation rate in the virtual $\Gamma_{c}$ states and resulting local photocurrent spectrum in the $X$-valley band at a photon energy $E_{\text {phot }}=1.17 \mathrm{eV}$ and very low internal field $F=2 \mathrm{kV} / \mathrm{cm}$. The generation

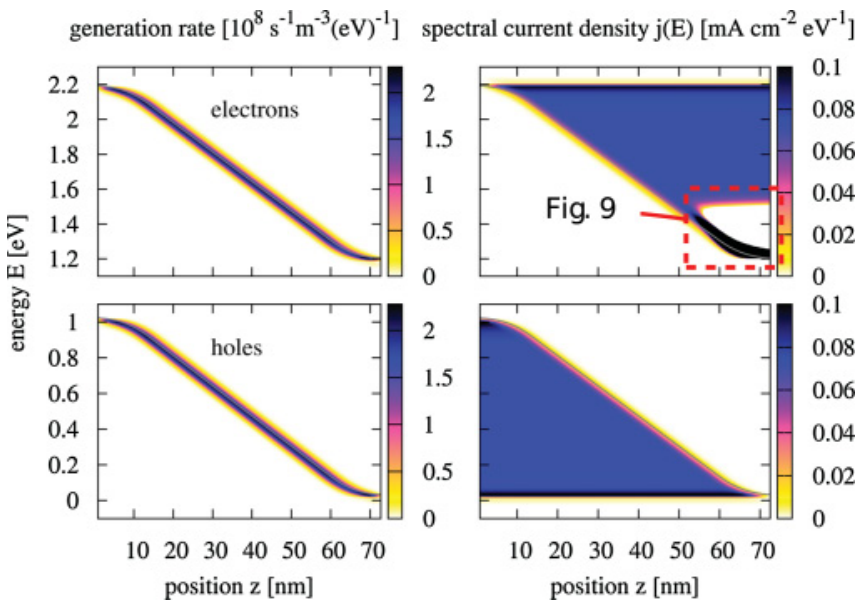

FIG. 8. (Color online) Same as in Fig. 7 but for a strong internal field of $F=169 \mathrm{kV} / \mathrm{cm}$. The large deviation close to the $n$-contact is due to the electron-phonon intraband scattering current that vanishes on energy integration over the band (white = negative, black = large positive; see Fig. 9). Due to the large field and the absence of additional, intraband scattering processes, the photocurrent is quasiballistic.

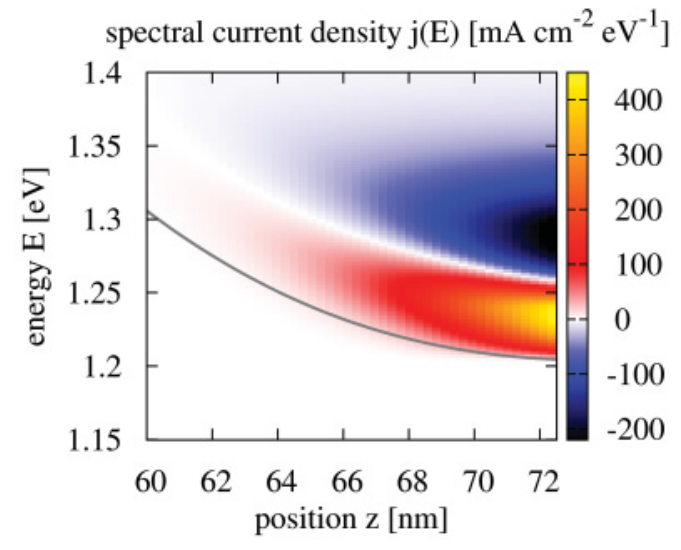

FIG. 9. (Color online) Current spectrum of $X$-valley electrons close to the $n$-contact. Due to the high electron density in this region, the spectral scattering current due to electron-phonon coupling is large but vanishes on integration over energy, i.e., there is no net transport in the $z$ direction due to electron-phonon scattering.

is relatively uniform throughout the device, as well as the increase of electron and hole current components toward the respective contacts. Figure 8 illustrates the corresponding situation for the diode with strong internal field $F=169 \mathrm{kV} / \mathrm{cm}$. In this case, the photogeneration and current contributions are distributed over a large energy range, the latter due to the absence of efficient (intraband) relaxation mechanisms that would confine current flow closer to the bandedge, resulting in a quasiballistic photocurrent. In the vicinity of the $n$-contact, the high electron concentration results in large effective electron-phonon intraband scattering current contributions (Fig. 9), which are mediated by the $\Gamma_{c}-X$ interband scattering mechanism and, due to the opposite signs of in- and outscattering components, cancel on energy integration over the conduction bands. The net current can thus be attributed exclusively to interband transitions, and the local sum of electron and hole contributions is perfectly conserved, as evidenced by Fig. 10. Since the photogeneration rate is intimately related to the $\Gamma-X$ intervalley electron-phonon scattering rate via the self-consistent computation of Green's functions and self-energies, and the photocurrent results from photogenerated excess charge that is transferred via the

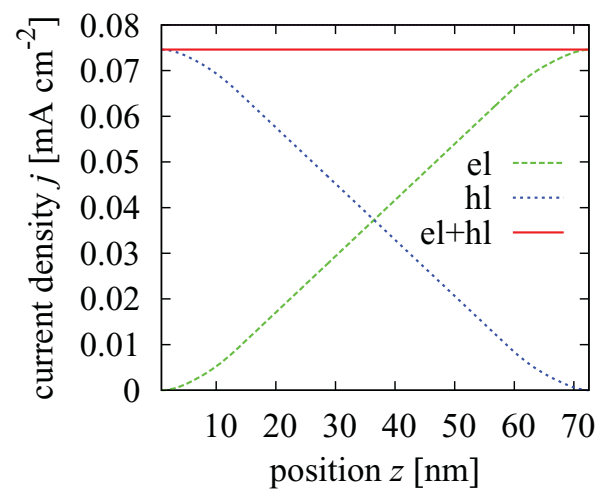

FIG. 10. (Color online) Integrated net current density for electrons (el) and holes (hl) in the high-field device. The total current, i.e., the sum of electron and hole current, is perfectly conserved over the whole device. 

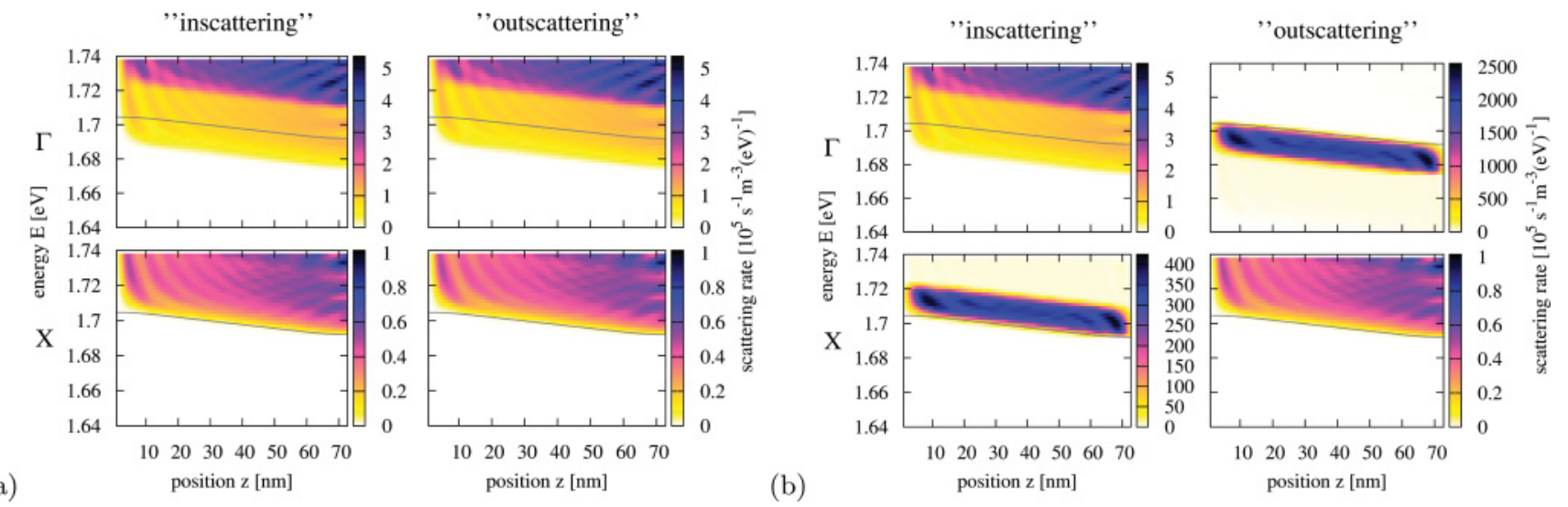

FIG. 11. (Color online) Phonon-mediated electron transfer rate between virtual $\left(\Gamma_{c}\right)$ and extended $(X)$ conduction band states (a) in the dark and (b) under illumination with $E_{\text {phot }}=1.17 \mathrm{eV}$, for the low-doping diode, where the intrinsic region extends over the whole device. While in- and outscattering are balanced in the dark, there is a strong net charge transfer from $\Gamma_{c}$ to $X$ under illumination, and the inscattering rate reflects the spectral pattern of the photogeneration.

scattering process with phonons to the extended $X$-valley states, the rates of this phonon-mediated charge transfer are expected to show the signature of the photogeneration process. Indeed, if the rate of electron scattering out of (into) the virtual $\Gamma_{c}$ states into (out of) the $X$ states is considered, there is a large net scattering from $\Gamma_{c}$ to $X$ under illumination, while in- and outscattering rates are balanced in the dark. This is shown in Fig. 11 for the case of the low-doping diode where the intrinsic region extends over the entire device. In the case of strong doping, the electron-phonon scattering rate in the dark is much stronger close to the contacts such that the effect becomes visible only in the central, intrinsic region of the junction. There, the phonon-related charge transfer rate again reflects the local photogeneration rate, as displayed in Fig. 12.

\section{CONCLUSIONS}

We have presented a novel theoretical approach to the description of phonon-mediated photogeneration in indirect semiconductors. While compatible with the standard Fermigolden rule approach in the quasiequilibrium bulk limit, its range of validity extends to quantum transport in open systems involving arbitrary heterostructure states far from equilibrium and the effects of nonlocality in the scattering processes, which are important aspects of advanced photovoltaic and light emitting devices, where often spatial and spectral resolution is required to gain access to a deeper understanding of the device characteristics. Thanks to this versatility, the theoretical framework lends itself to the modeling of indirect semiconductor based nanostructures with potential applications in a multitude of optoelectronic devices, such as silicon-based quantum well solar cells.

\section{ACKNOWLEDGMENTS}

Financial support was provided by the German Federal Ministry of Education and Research (BMBF) under Grant No. 03SF0352E.

\section{APPENDIX: INTERACTION SELF-ENERGIES}

The self-energies as defined by the Dyson equations (8) and (9) encode the renormalization of the charge carrier Green's
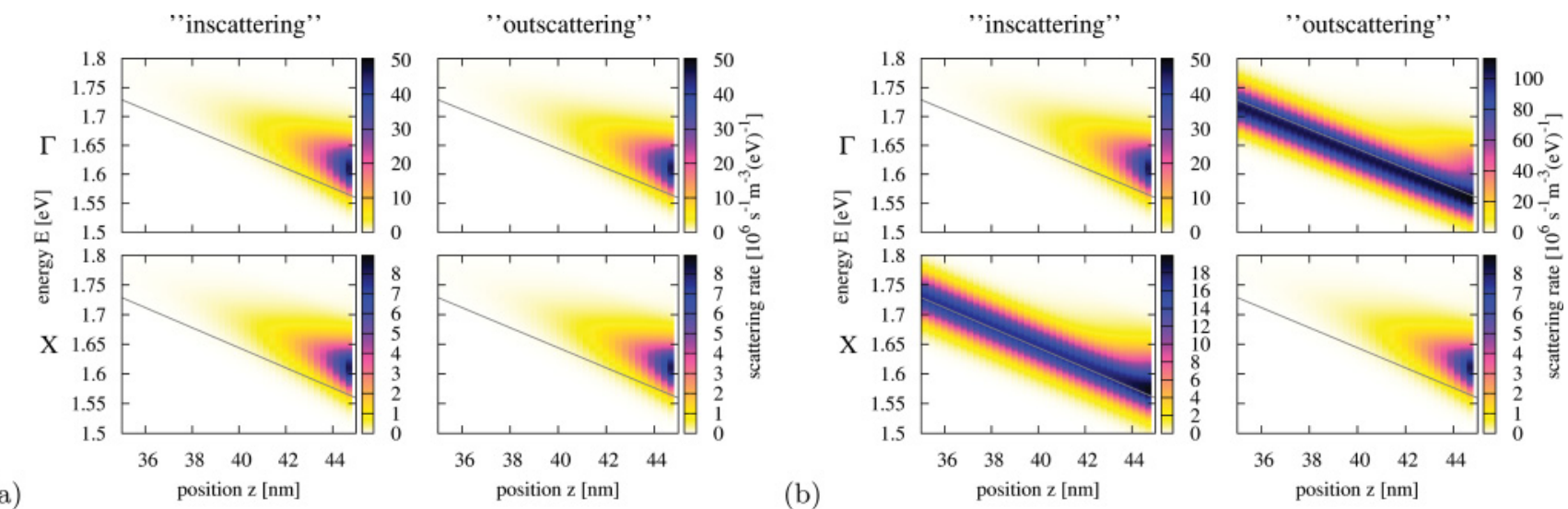

FIG. 12. (Color online) Phonon-mediated electron transfer rate between virtual and extended conduction band states (a) in the dark and (b) under illumination with $E_{\text {phot }}=1.17 \mathrm{eV}$ in the center of the short intrinsic region of the high-doping diode. Closer to the contacts, the scattering rate contributed by the photogeneration is masked by the large intraband relaxation rate. 
functions due to the interactions with photons and phonons, i.e., generation, recombination, and relaxation processes. The self-energies due to interactions can be evaluated from the perturbation expansion of the nonequilibrium statistical average defining the interacting NEGF, using either Wick's theorem or Feynman diagrams. In the first case, the (contour-ordered) self-energy $\Sigma$ is derived from a perturbation expansion of the exponential in the definition of the contour-ordered Green's function as the nonequilibrium expectation value of singlecarrier operators,

$$
G_{\alpha, \alpha^{\prime}}\left(\mathbf{k} ; t, t^{\prime}\right) \equiv-\frac{i}{\hbar}\left\langle\hat{T}_{C}\left\{e^{-\frac{i}{\hbar} \int_{C} d s H^{\prime}(s)} \hat{c}_{\alpha, \mathbf{k}}(t) \hat{c}_{\alpha^{\prime}, \mathbf{k}}^{\dagger}\left(t^{\prime}\right)\right\}\right\rangle
$$

$\alpha=n$ (band index) and $\mathbf{k}=\left(k_{x}, k_{y}, k_{z}\right)$ (bulk) or $\alpha=i, n$ and $\mathbf{k}=\left(k_{x}, k_{y}\right) \equiv \mathbf{k}_{\|}$(thin film). In the following, the Hamiltonian terms $H^{\prime}$ for the perturbative interaction of electrons with photons and phonons shall be discussed and used in the derivation of the corresponding self-energy expressions for bulk and thin-film systems.

\section{Interaction Hamiltonian}

a. Electron-photon interaction. For the electron-photon interaction, the perturbation Hamiltonian is given via the linear coupling to the vector potential operator of the electromagnetic field $\hat{\mathbf{A}}$,

$$
\hat{H}_{e \gamma}=-\frac{e}{m_{0}} \hat{\mathbf{A}} \cdot \hat{\mathbf{p}}
$$

with $\hat{\mathbf{p}}$ the momentum operator and

$$
\hat{\mathbf{A}}(\mathbf{r}, t)=\sum_{\lambda, \mathbf{q}}\left[\mathbf{A}_{0}(\lambda, \mathbf{q}) \hat{a}_{\lambda, \mathbf{q}}(t)+\mathbf{A}_{0}^{*}(\lambda,-\mathbf{q}) \hat{a}_{\lambda,-\mathbf{q}}^{\dagger}(t)\right] e^{i \mathbf{q r}},
$$

$$
\mathbf{A}_{0}(\lambda, \mathbf{q})=\frac{\hbar}{\sqrt{2 \epsilon_{0} V \hbar \omega_{\lambda \mathbf{q}}}} \boldsymbol{\epsilon}_{\lambda \mathbf{q}}
$$

where $\boldsymbol{\epsilon}_{\lambda \mathbf{q}}$ is the polarization of the photon with wave vector $\mathbf{q}$ and energy $\hbar \omega_{\lambda \mathbf{q}}$ added to or removed from photon mode $(\lambda, \mathbf{q})$ by the bosonic creation and annihilation operators

$$
\hat{a}_{\lambda, \mathbf{q}}^{\dagger}(t)=\hat{a}_{\lambda, \mathbf{q}}^{\dagger} e^{i \omega_{\lambda \mathbf{q}} t}, \quad \hat{a}_{\lambda, \mathbf{q}}(t)=\hat{a}_{\lambda, \mathbf{q}} e^{-i \omega_{\lambda \mathbf{q}} t},
$$

and $V$ is the absorbing volume.

For numerical evaluation, the Hamiltonian is reformulated in a suitable representation using the field operators given in (10) (bulk) and (46) (thin film), respectively,

$$
\mathcal{H}_{e \gamma}(t)=\int d^{3} r \hat{\Psi}^{\dagger}(\mathbf{r}, t) \hat{H}_{e \gamma} \hat{\Psi}(\mathbf{r}, t) .
$$

In the bulk case, the above expression results in ${ }^{24}$

$$
\begin{aligned}
\mathcal{H}_{e \gamma}(t)= & \sum_{\mathbf{q}, \lambda} \sum_{n, m} \sum_{\mathbf{k}, \mathbf{k}^{\prime}} \mathcal{M}_{n, m}^{\gamma \gamma}\left(\mathbf{k}, \mathbf{k}^{\prime}, \mathbf{q}, \lambda\right) \\
& \times \hat{c}_{n \mathbf{k}}^{\dagger}(t) \hat{c}_{m \mathbf{k}^{\prime}}(t)\left[\hat{a}_{\lambda, \mathbf{q}} e^{-i \omega_{\lambda \mathbf{q}} t}+\hat{a}_{\lambda,-\mathbf{q}}^{\dagger} e^{i \omega_{\lambda \mathbf{q}} t}\right],
\end{aligned}
$$

where the matrix element for interband transitions $(n \neq m)$ is obtained from a $\mathbf{k} \cdot \mathbf{p}$-type approximation, ${ }^{11}$

$$
\begin{aligned}
\mathcal{M}_{n, m}^{e \gamma}\left(\mathbf{k}, \mathbf{k}^{\prime}, \mathbf{q}, \lambda\right) \equiv & -\frac{e}{m_{0}} A_{0}(\lambda, \mathbf{q}) \\
& \times \int d^{3} r \psi_{n \mathbf{k}}^{*}(\mathbf{r})\left(e^{i \mathbf{q r}} \boldsymbol{\epsilon}_{\lambda \mathbf{q}} \cdot \hat{\mathbf{p}}\right) \psi_{m \mathbf{k}^{\prime}}(\mathbf{r}) \\
\approx & -\frac{e}{m_{0}} A_{0}(\lambda, \mathbf{q}) \delta\left(\mathbf{k}^{\prime}+\mathbf{q}-\mathbf{k}\right)\left(\boldsymbol{\epsilon}_{\lambda \mathbf{q}} \cdot \mathbf{p}_{n m}\right),
\end{aligned}
$$

with the Bloch function momentum matrix element

$$
\mathbf{p}_{n m}=\int_{\Omega} \frac{d^{3} \tilde{r}}{\Omega} u_{n \mathbf{k}_{0}}(\tilde{\mathbf{r}}) \hat{\mathbf{p}} u_{m \mathbf{k}_{0}^{\prime}}(\tilde{\mathbf{r}}),
$$

where $\Omega$ denotes the unit-cell volume. This gives the final bulk expression

$$
\begin{aligned}
\mathcal{H}_{e \gamma}(t)= & \sum_{\mathbf{q}, \lambda} \sum_{n, m} \sum_{\mathbf{k}} \mathcal{M}_{n, m}^{e \gamma}(\mathbf{k}, \mathbf{q}, \lambda) \\
& \times \hat{c}_{n \mathbf{k}}^{\dagger}(t) \hat{c}_{m \mathbf{k}}(t)\left[\hat{a}_{\lambda, \mathbf{q}} e^{-i \omega_{\lambda \mathbf{q}} t}+\hat{a}_{\lambda,-\mathbf{q}}^{\dagger} e^{i \omega_{\lambda \mathbf{q}} t}\right] .
\end{aligned}
$$

For devices with broken translational invariance in the transport dimension, the representation of the electron-photon Hamiltonian (A2) in the real-space effective mass basis (47) acquires the similar from

$$
\begin{aligned}
\mathcal{H}_{e \gamma}(t)= & \sum_{\mathbf{q}, \lambda} \sum_{i n, j m} \sum_{\mathbf{k}_{\|}} \mathcal{M}_{i n, j m}^{e \gamma}\left(\mathbf{k}_{\|}, \mathbf{q}, \lambda\right) \\
& \times \hat{c}_{i n \mathbf{k}_{\|}}^{\dagger}(t) \hat{c}_{j m \mathbf{k}_{\|}}(t)\left[\hat{a}_{\lambda, \mathbf{q}} e^{-i \omega_{\lambda \mathbf{q}} t}+\hat{a}_{\lambda,-\mathbf{q}}^{\dagger} e^{i \omega_{\lambda \mathbf{q}} t}\right],
\end{aligned}
$$

where

$$
\mathcal{M}_{i n, j m}^{e \gamma}\left(\mathbf{k}_{\|}, \mathbf{q}, \lambda\right) \approx-\frac{e}{m_{0}} A_{0}(\lambda, \mathbf{q}) M_{i j}\left(q_{z}\right)\left(\boldsymbol{\epsilon}_{\lambda \mathbf{q}} \cdot \mathbf{p}_{n m}\right),
$$

with

$$
M_{i j}\left(q_{z}\right)=\int d z \chi_{i}^{*}(z) e^{i q_{z} z} \chi_{j}(z)=e^{i q_{z} z_{i}} \delta_{i j}
$$

Electron-phonon interaction. The vibrational degrees of freedom of the system are described in terms of the coupling of the force field of the electron-ion potential $V_{\mathrm{ei}}$ to the quantized field $\hat{\mathcal{U}}$ of the ionic displacement, ${ }^{25}$

$$
\hat{H}_{\mathrm{ep}}(\mathbf{r}, t)=\sum_{\mathbf{L}, \boldsymbol{\kappa}} \hat{\mathcal{U}}(\mathbf{L}+\boldsymbol{\kappa}, t) \cdot \nabla V_{\mathrm{ei}}[\mathbf{r}-(\mathbf{L}+\boldsymbol{\kappa})],
$$

with the displacement field given by the Fourier expansion

$$
\begin{aligned}
\hat{\mathcal{U}}_{\alpha}(\mathbf{L} \kappa, t)= & \sum_{\Lambda, \mathbf{Q}} \mathcal{U}_{\alpha \kappa}(\Lambda, \mathbf{Q}) e^{i \mathbf{Q} \cdot(\mathbf{L}+\kappa)}\left[\hat{b}_{\Lambda, \mathbf{Q}}(t)+\hat{b}_{\Lambda,-\mathbf{Q}}^{\dagger}(t)\right], \\
& (\alpha=x, y, z),
\end{aligned}
$$

where the ion equilibrium position is $\mathbf{L}+\boldsymbol{\kappa}$, with $\mathbf{L}$ the lattice position and $\boldsymbol{\kappa}$ the relative position of a specific basis atom at this lattice site, and $\hat{b}_{\Lambda, \mathbf{Q}}, \hat{b}_{\Lambda, \mathbf{Q}}^{\dagger}$ are the bosonic creation and annihilation operators for a (bulk) phonon mode with polarization $\Lambda$ and wave vector $\mathbf{Q}$ in the first Brillouin zone. 
The potential felt by electrons in heterostructure states due to coupling to bulk phonons can thus be written as

$$
\hat{H}_{\mathrm{ep}}(\mathbf{r}, t)=\frac{1}{\sqrt{V}} \sum_{\Lambda \mathbf{Q}} U_{\Lambda, \mathbf{Q}} e^{i \mathbf{Q} \cdot \mathbf{r}}\left\{\hat{b}_{\Lambda, \mathbf{Q}}(t)+\hat{b}_{\Lambda,-\mathbf{Q}}^{\dagger}(t)\right\}
$$

where $\mathbf{r}$ is the electron coordinate, and $U_{\Lambda, \mathbf{Q}}$ are related to the Fourier coefficients of the electron-ion potential. ${ }^{26}$

The effective-mass Hamiltonian for electron-phonon interaction is obtained from (A17) in analogy to the electron-photon interaction, with the bulk result

$$
\begin{aligned}
\mathcal{H}_{\mathrm{ep}}(t)= & \sum_{\mathbf{Q}, \Lambda} \sum_{n, \mathbf{k}} \mathcal{M}^{\mathrm{ep}}(\mathbf{Q}, \Lambda) \hat{c}_{n \mathbf{k}}^{\dagger}(t) \hat{c}_{n \mathbf{k}-\mathbf{Q}}(t) \\
& \times\left[\hat{b}_{\Lambda, \mathbf{Q}} e^{-i \Omega_{\Lambda, \mathbf{Q}} t}+\hat{b}_{\Lambda,-\mathbf{Q}}^{\dagger} e^{i \Omega_{\Lambda, \mathbf{Q}} t}\right],
\end{aligned}
$$

with

$$
\mathcal{M}^{\mathrm{ep}}(\mathbf{Q}, \Lambda)=\frac{U_{\Lambda, \mathbf{Q}}}{\sqrt{V}},
$$

and the thin-film expression

$$
\begin{aligned}
\mathcal{H}_{\mathrm{ep}}(t)= & \sum_{\mathbf{Q}, \Lambda} \sum_{n} \sum_{\mathbf{k}_{\|}, i} \mathcal{M}_{i}^{\mathrm{ep}}(\mathbf{Q}, \Lambda) \hat{c}_{i n \mathbf{k}_{\|}^{\dagger}}^{\dagger}(t) \hat{c}_{i n \mathbf{k}_{\|}-\mathbf{Q}_{\|}(t)} \\
& \times\left[\hat{b}_{\Lambda, \mathbf{Q}} e^{-i \Omega_{\Lambda, \mathbf{Q}} t}+\hat{b}_{\Lambda,-\mathbf{Q}}^{\dagger} e^{i \Omega_{\Lambda, \mathbf{Q}} t}\right],
\end{aligned}
$$

where

$$
\mathcal{M}_{i}^{\mathrm{ep}}(\mathbf{Q}, \Lambda)=\frac{U_{\Lambda, \mathbf{Q}}}{\sqrt{V}} e^{i Q_{z} z_{i}} .
$$

For the $\Gamma-X$ intervalley scattering considered in the present discussion, the coupling reads

$$
\left|U_{\Lambda, \mathbf{Q}}\right|^{2}=\frac{\hbar\left(D_{i v} K\right)_{\sigma}^{2}}{2 \rho \Omega_{\sigma}},
$$

where $\sigma$ labels the phonon mode, $D_{i v}$ is the associated deformation potential, and $K$ denotes the momentum transfer required for the scattering between two valleys.

\section{a. Self-energy}

At this stage, any renormalizing effect of the electronic system on the photons and phonons is neglected, i.e., the coupling to the bosons corresponds to the connection to corresponding equilibrium reservoirs. While this treatment is generally a good approximation in the case of phonons, it is valid for the coupling to the photonic systems only in the case of low absorption, i.e., weak coupling or very short absorber length.
The equilibrium propagators for noninteracting photons and phonons in isotropic media have the common form $(\alpha=\gamma, p h)$

$$
\begin{gathered}
D_{\lambda}^{\alpha, \lessgtr}(\mathbf{q} ; E)=-2 \pi i\left[N_{\lambda, \mathbf{q}}^{\alpha} \delta\left(E \mp \hbar \omega_{\lambda \mathbf{q}}\right)\right. \\
\left.+\left(N_{\lambda, \mathbf{q}}^{\alpha}+1\right) \delta\left(E \pm \hbar \omega_{\lambda \mathbf{q}}\right)\right], \\
D_{\lambda}^{\alpha, R / A}(\mathbf{q} ; E)=\frac{1}{E-\hbar \omega_{\lambda \mathbf{q}} \pm i \eta}-\frac{1}{E+\hbar \omega_{\lambda \mathbf{q}} \pm i \eta} .
\end{gathered}
$$

In the above expressions, $N_{\lambda, \mathbf{q}}^{\alpha}$ denotes the occupation of the respective equilibrium boson modes, with the phonon occupation given by the Bose-Einstein distribution at lattice temperature $T$,

$$
N_{\Lambda, \mathbf{Q}}^{p h}=\left(e^{\beta \hbar \Omega_{\Lambda, \mathbf{Q}}}-1\right)^{-1}, \quad \beta=\left(k_{B} T\right)^{-1},
$$

and the photon occupation $N_{\lambda, \mathbf{q}}^{\gamma}$ introduced in Sec. II B 1. Inserting these propagators the Fock-term of the a general electron-boson self-energy in the first self-consistent Born approximation provides the steady-state components $(\alpha=$ $\gamma, p h)$

$$
\begin{aligned}
& \boldsymbol{\Sigma}_{e \alpha}^{\lessgtr}(\mathbf{k} ; E)=\sum_{\lambda, \mathbf{q}} \mathcal{M}^{e \alpha}(\mathbf{k}, \mathbf{q}, \lambda)\left[N_{\lambda, \mathbf{q}}^{\alpha} \mathbf{G}^{\lessgtr}\left(\mathbf{k} ; E \mp \hbar \omega_{\lambda, \mathbf{q}}\right)\right. \\
& \left.+\left(N_{\lambda, \mathbf{q}}^{\alpha}+1\right) \mathbf{G}^{\lessgtr}\left(\mathbf{k} ; E \pm \hbar \omega_{\lambda, \mathbf{q}}\right)\right] \\
& \times \mathcal{M}^{e \alpha}(\mathbf{k},-\mathbf{q}, \lambda)
\end{aligned}
$$

and

$$
\Sigma_{e \alpha}^{R, A}(\mathbf{k} ; E)=i \int \frac{d E^{\prime}}{2 \pi} \frac{\Sigma_{e \alpha}^{>}\left(\mathbf{k} ; E^{\prime}\right)-\Sigma_{e \alpha}^{<}\left(\mathbf{k} ; E^{\prime}\right)}{E^{\prime}-E \pm i \eta}
$$

with the same definitions of indices and momentum as in (A1). Using a constant mode-specific coupling strength, the intervalley bulk (thin-film) electron-phonon scattering selfenergy is further simplified to the diagonal form used in the simulations,

$$
\begin{aligned}
\Sigma_{(i j,) b}^{\lessgtr}(E)= & \sum_{\sigma} \sum_{b^{\prime} \neq b} \frac{\hbar\left(D_{i v} K\right)_{\sigma}^{2}}{2 \rho \Omega_{\sigma} \Delta} f_{b^{\prime}}\left(\delta_{i, j}\right) \\
& \times \int \frac{d \mathbf{k}}{(2 \pi)^{d}}\left[N_{\sigma}^{p h} G_{(i j,) b^{\prime}}^{\lessgtr}\left(\mathbf{k} ; E \pm \hbar \Omega_{\sigma}\right)\right. \\
& \left.+\left(N_{\sigma}^{p h}+1\right) G_{(i j,) b^{\prime}}^{\lessgtr}\left(\mathbf{k} ; E \mp \hbar \Omega_{\sigma}\right)\right], \quad(
\end{aligned}
$$

where $b, b^{\prime} \in\left\{\Gamma_{c}, X\right\}$ and $d=2$ (thin film) or 3 (bulk). *u.aeberhard@fz-juelich.de

${ }^{1}$ M. A. Green, J. Mater. Sci. Eng. B 74, 118 (2000).

${ }^{2}$ M. A. Green, Prog. Photovolt: Res. Appl. 9, 123 (2001).

${ }^{3}$ C. Delerue, G. Allan, and M. Lannoo, Phys. Rev. B 64, 193402 (2001).

${ }^{4}$ U. Aeberhard and R. H. Morf, Phys. Rev. B 77, 125343 (2008).

${ }^{5}$ K. Henneberger and H. Haug, Phys. Rev. B 38, 9759 (1988).

${ }^{6}$ F. Jahnke and S. W. Koch, Phys. Rev. A 52, 1712 (1995).

${ }^{7}$ K. Henneberger and S. W. Koch, Phys. Rev. Lett. 76, 1820 (1996).
${ }^{8}$ M. F. Pereira and K. Henneberger, Phys. Rev. B 53, 16485 (1996).

${ }^{9}$ M. F. Pereira and K. Henneberger, Phys. Rev. B 58, 2064 (1998).

${ }^{10} \mathrm{~K}$. Henneberger and F. Richter, Phys. Rev. A 80, 013807 (2009).

${ }^{11}$ S. Steiger, Ph.D. thesis, ETH Zurich, 2009.

${ }^{12} \mathrm{~S}$. Steiger, R. G. Veprek, and B. Witzigmann, in Proceedings of the 2009 13th International Workshop on Computational Electronics, IWCE 2009 (IWCE, New York, 2009).

${ }^{13}$ The dimensions are that of a volume rate, $[\mathcal{G}, \mathcal{R}]=m^{-3} s^{-1}$. 
${ }^{14}$ L. P. Kadanoff and G. Baym, Quantum Statistical Mechanics (Benjamin, Reading, MA, 1962).

${ }^{15}$ L. Keldysh, Sov. Phys.-JETP 20, 1018 (1965).

${ }^{16}$ The factor of 2 is due to the assumption of spin degeneracy.

${ }^{17}$ F. Bassani and G. Pastori Parravicini, Electronic States and Optical Transitions in Solids, edited by R. A. Ballinger (Pergamon Press, Oxford, 1975).

${ }^{18}$ T. Kubis, C. Yeh, P. Vogl, A. Benz, G. Fasching, and C. Deutsch, Phys. Rev. B 79, 195323 (2009).

${ }^{19}$ R. Lake, G. Klimeck, R. Bowen, and D. Jovanovic, J. Appl. Phys. 81, 7845 (1997)

${ }^{20}$ L. E. Henrickson, J. Appl. Phys 91, 6273 (2002).
${ }^{21}$ S. Jin, Ph.D. thesis, School of Electrical Engineering and Computer Science College of Engineering, Seoul National University, 2006.

${ }^{22}$ U. Aeberhard, Nanoscale Res. Lett. 6, 242 (2011).

${ }^{23}$ For an explicit derivation of the contact self-energy in the effectivemass tight-binding model see, e.g., Ref. 19.

${ }^{24}$ The photon momentum is neglected as compared to the electron quasimomentum.

${ }^{25} \mathrm{~W}$. Schäfer and M. Wegener, Semiconductor Optics and Transport Phenomena (Springer, Berlin, 2002).

${ }^{26}$ G. D. Mahan, Many-Particle Physics, 2nd ed. (Plenum, New York, 1990). 